\title{
Review Article \\ The WArP Experiment: A Double-Phase Argon Detector for Dark Matter Searches
}

\author{
Andrea Zani \\ Pavia University and INFN Pavia, Via Bassi 6, 27100 Pavia, Italy \\ Correspondence should be addressed to Andrea Zani; andrea.zani@pv.infn.it
}

Received 5 December 2013; Accepted 19 February 2014; Published 30 April 2014

Academic Editor: Claudio Montanari

Copyright (C) 2014 Andrea Zani. This is an open access article distributed under the Creative Commons Attribution License, which permits unrestricted use, distribution, and reproduction in any medium, provided the original work is properly cited. The publication of this article was funded by SCOAP ${ }^{3}$.

\begin{abstract}
Cryogenic noble liquids emerged in the previous decade as one of the best media to perform WIMP dark matter searches, in particular due to the possibility to scale detector volumes to multiton sizes. The WArP experiment was then developed as one of the first to implement the idea of coupling Argon in liquid and gas phase, in order to discriminate $\beta / \gamma$-interactions from nuclear recoils and then achieve reliable background rejection. Since its construction, other projects spawned, employing Argon and Xenon and following its steps. The WArP 100l detector was assembled in 2008 at the Gran Sasso National Laboratories (LNGS), as the final step of a years-long R\&D programme, aimed at characterising the technology of Argon in double phase for dark matter detection. Though it never actually performed a physics run, a technical run was taken in 2011, to characterise the detector response.
\end{abstract}

\section{Introduction}

Recent results on cosmic microwave background by the Planck space observatory have brought further confirmation that an important component of our Universe comes in the form of nonbaryonic dark matter, with an abundance far higher than that of known baryonic matter. According to the latest Planck results, it is $\Omega_{\mathrm{DM}} \simeq 26.8 \%$ and $\Omega_{B} \simeq 4.9 \%[1,2]$.

Several theories have been developed in the past to describe the nature of dark matter (DM) [3], and one interesting class of candidates is the so-called weakly interactive massive particles (WIMPs). In the past years the preferred candidate of this class of theories came from supersymmetric extensions of the Standard Model [4]. According to these models, experiments on Earth surface can detect elastic scatterings on target nuclei by crossing WIMPs that are part of the dark halo surrounding our Galaxy [5]. The energies of the recoiling nuclei should range from few to a hundred $\mathrm{keV}$. The cross sections and rates depend on many parameters: the chosen WIMP candidate and nuclear model, the target material, and the distribution of velocities of the DM particles in the halo surrounding our galaxy. The variety of theories implies that allowed regions by theoretical models in the parameter space, characterised by the mass $(m)$ and WIMPnucleon cross section $(\sigma)$ of these particles, span many orders of magnitude.

So far, most experiments have tried to detect the signatures of the interaction, in the form of light, charge, or heat. More recent experiments choose to detect simultaneously two signatures, which allow performing particle discrimination and rejecting backgrounds not due to nuclear recoils. Most recent negative results come from XENON100 (2012) [6, 7] and LUX (2013) [8], which pushes the limit on spin-independent, WIMP-nucleon cross section below $10^{-45} \mathrm{~cm}^{2}$. This is in contrast with experiments searching for a seasonal modulation in the spectrum of recoils, which is ascribed to the Earth variation of relative velocity with respect to the DM halo, during its revolution around the Sun. The DAMA [9] and CoGeNT [10, 11] Collaborations reported positive detection of an annual modulation. Only one experiment searching for a double interaction signature (in this case light emission and heat depositions) reported a positive result as well, that is, the CRESST-II experiment in Gran Sasso [12]. A definitive answer to the identification 
of dark matter is however yet to come, as the allowed regions in the parameters spaces, selected by these experiments, were all excluded by mentioned LUX latest data [8].

The WArP (Wimp Argon Programme) experiment represents one of the first attempts to use liquefied noble gases as target for the interaction. The use of noble liquids implies the need to operate at cryogenic temperatures (87 K Argon, $165 \mathrm{~K}$ Xenon), but they behave as scintillators, which means that any ionising particle in the medium produces both light and charge. The implementation of the double-phase (described in the next section) is then introduced, to exploit simultaneously the charge/light signatures and to probe the low recoil-energy range predicted by the theories. The WArP Collaboration carried on years of studies on liquid Argon and double-phase technologies: this led to the operation of a 2.31 prototype (Section 3.1), which successfully tested the new detection technique, and to the implementation of a full-scale 1001 detector, aimed at dark matter searches (Section 3.2).

\section{Liquid Argon}

The WArP technology was developed through years of R\&D studies, started at CERN in the 1990s, as part of the activities related to the ICARUS experiment [13]. First studies to discriminate between nuclear recoils and $\beta / \gamma$-induced events were performed in liquid Xenon [14], but later the decision was made to employ Argon instead, coupled to the concept of a double-phase chamber [15].

Noble liquids are scintillators, with energies of the order of tens of eV required to produce a scintillation photon. For example, a relativistic electron needs to deposit about $20 \mathrm{eV}$ in Argon to produce a scintillation photon; the value increases for heavier, slower particles. First tests were performed on Xenon: the reason is that it has slightly better physical properties with respect to Argon, like higher electron mobility, density, and boiling point. On the other hand, Argon is more commonly found in atmosphere, and state-of-the-art technology to obtain, store, and purify it is already available and cheap. This makes it possible to scale detectors up to multikton volumes.

A further physical significant advantage of Argon over Xenon is related to light emission. Scintillation light in Argon is emitted in the vacuum ultraviolet (VUV) region, at $128 \mathrm{~nm}$, from deexcitation of $\mathrm{Ar}_{2}^{*}$ dimers. These can be produced either by excitation of the medium, or following recombination after production of electron-ion pairs. The energy required to produce a pair is dependent on the nature of the interacting particle. For a wide range of particles, from relativistic ions and electrons to $\alpha$ 's, it is measured to be $23.6 \mathrm{eV}[16,17]$. The value increases for heavy slow ion recoils $(\beta<0.01)$, due to the decreasing efficiency of the ionization process. The description of the mechanism in this energy range was first suggested by Lindhard et al. [18, 19]. The first experimental confirmations of the theory came in the $1960 \mathrm{~s}$ $[20,21]$; later, the WArP collaboration tested independently the model for the case of Argon nuclei recoiling in LAr [22], confirming its validity.
TABLE 1: Relative fast-to-slow signal intensity ratio in LAr for different particles in zero-field conditions [23]. LET increases from electrons to fission fragments, and $I_{f} / I_{s}$ increases as well.

\begin{tabular}{lc}
\hline Ionising particle & $I_{f} / I_{s}$ \\
\hline Rel. electrons & 0.3 \\
$\alpha$-Particles & 1.3 \\
Fission fragments & 3.0 \\
\hline
\end{tabular}

In general, the amount of produced scintillation light and ionization depends on the way particles deposit their energy in the medium, that is, the Linear Energy Transfer (LET). Therefore, both light and charge can be used to perform particle identification, based on the characteristics of the collected signals.

Both luminescence processes discussed above are characterised by two components with different decay times. The fast one has a decay-constant $\tau_{f} \approx 6 \mathrm{~ns}$, while the other is far slower, with $\tau_{s} \approx 1200-1500 \mathrm{~ns}$. Such a large difference in the values of the time constants is related to the characteristics of the excited states in which the Argon dimers appear, and it shows no dependence on the particle LET. However, it has been demonstrated [23] that it is the relative intensity of the two components (fast-to-slow, $I_{f} / I_{s}$ ) to be heavily dependent on LET, with their ratio increasing with it (see Table 1). Therefore, different particles produce light signals characterised by different shapes and profiles as a function of time: in particular electrons are expected to produce signals dominated by the slow light component, while $\alpha$ 's and nuclear recoils, characterised by a higher LET, induce much faster signals. An analysis of the pulse shape, applied to the collected signals, allows recognising distinct particles interacting in LAr, therefore providing a powerful method to reject backgrounds. As mentioned, this is made possible only by the fact that Argon light decay constants are very well separated in magnitude: the same is not applicable to, for example, Xenon, which has both decay-time constants in range of few ns-few tens of ns.

A drawback of Argon is the contamination of ${ }^{39}$ Ar. It is a $\beta^{-}$-emitter with half-life of $269 \mathrm{y}$ and end-point at $565 \mathrm{keV}$; its activity was measured by the WArP Collaboration as $(1.01 \pm 0.08) \mathrm{Bq}$ per $\mathrm{kg}$ of natural Argon, corresponding to a concentration of $(8.0 \pm 0.6) \cdot 10^{-16} \mathrm{~g}\left({ }^{39} \mathrm{Ar}\right) / \mathrm{g}\left({ }^{\text {nat }} \mathrm{Ar}\right)[24]$.

${ }^{39} \mathrm{Ar}$ is produced by interaction of cosmic rays with the atmosphere, and it is naturally present in commercial Argon; however, it was recently discovered [25] that underground Argon pockets contain very low levels of ${ }^{39} \mathrm{Ar}$, due to their isolation from Earth surface and atmospheric radiation. The extraction technology is not yet perfected, and costs are high, therefore this solution is still not any more convenient than using liquid Xenon.

The choice of the target material is also dependent on the need to enhance as much as possible the interaction rate of WIMPs, characterised by weak cross sections. The integrated cross section for elastic scatterings on a nucleus is proportional to $A^{2}$, with $A$ being the mass number, due to the coherence effect. Then Argon is expected to have 


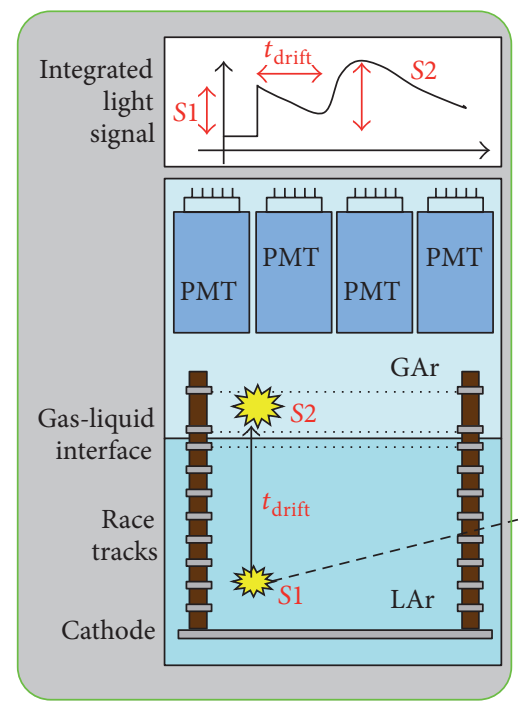

FIGURE 1: Working scheme of the inner detector.

a lower integrated cross section, with respect to "heavier" materials like Xenon or Germanium; however, the spectra of these elements are suppressed at higher energies by the nuclear form factor [22]. Then, by assuming a low recoil energy threshold at around $30 \mathrm{keV}$, the integrated rates of the considered materials will be similar in magnitude.

In conclusion, taking into account the availability at cheap cost of large quantities of highly purified Argon, the expected rates of interaction, and its scintillation light properties, LAr can be considered as a preferred medium for future largevolume dark matter experiments.

2.1. Double Phase Technology. The characteristics of liquid Argon are coupled, for dark matter searches, to the innovative concept of a double-phase chamber ([15], see Figure 1), which derives from the time projection chamber (TPC) design [26].

In this new implementation, a gaseous Argon (GAr) pocket is coupled to a liquid volume, in which a uniform electric field is applied. Interactions taking place in the liquid produce both a primary scintillation light signal $(s 1)$ and ionization electrons. The light is collected by means of cryogenic photomultipliers (PMTs); the charge is drifted to the liquid surface by a uniform electric field. There, a grid system provides electron extraction into the gas phase and acceleration, in order to produce secondary light emission $(s 2)$, which is proportional to the initially produced charge. The amount of collected light (light yield, LY) is expressed in phe $/ \mathrm{keV}$, that is, number of collected photoelectrons per $\mathrm{keV}$ of energy deposited in the liquid.

As already discussed in Section 2, the relative intensity of primary scintillation and ionization is dependent on the particle nature; therefore, it is expected that distinct particles will produce different values of the ratio $s 2 / s 1$. The same can be stated for the fraction of fast component $(s f)$ present in the primary signal: once again this is dependent on which particle is interacting in LAr. These two features can be exploited to recognise particles, through the analysis of light signals shapes and intensities. Figure 2 depicts real events recorded with a prototype chamber used during the R\&D of the WArP project: it can be seen as in particular integrated signals show the difference between the response produced, following an interaction by a fast, light particle (electron, left) and a nuclear recoil (right). Different particles will produce distinct signal shapes and intensities, allowing performing particle identification, and thus rejection of events not due to nuclear recoils.

Numerous technical issues were to be investigated when the WArP Collaboration first came out with this solution, and the necessary activities were carried out by the various groups of the collaboration [27].

The processes of electron extraction and multiplication in the gas phase were investigated, along with the proportionality of secondary light to the emitted charge [28]. The solution eventually adopted within the collaboration consists in a system of three metallic wire grids independently fed. The first grid is put beneath the liquid surface, while the other two are in the gas volume. The region between the first and second grid is where extraction and acceleration are performed, by means of an electric field. The field region between the second and third grid is instead configured to collect all the electrons on the last plane, in order to protect the PMTs that are placed behind it. The study of the extraction/multiplication fields was carried on with a 2.31 prototype chamber [22].

The issue of light collection was also heavily investigated: first of all it is necessary to employ a wavelength shifter, in order to shift the scintillation VUV photons produced in Argon into the visible range. Then, as the detector is designed to have photomultipliers on a single side (facing the gas pocket), all other internal surfaces must be covered with a reflecting material, in order not to lose light. The solution chosen by the collaboration, after a series of tests performed both at CERN and LNGS, is to use VM2000, a dielectric reflector from $3 \mathrm{M}$ (not affected by the electric field), on which tetraphenyl-butadiene (TPB), that shifts VUV photons to $430 \mathrm{~nm}$, is deposited through vacuum evaporation. TPB is also deposited in a thin toluene film on the PMTs windows, so that all produced photons can undergo shifting before reaching the detectors. The choice of materials and of the method of deposition for TPB on VM2000 was achieved after dedicated tests carried out at CERN. Vacuum evaporation on all inner detector surfaces was chosen as it proved to be the deposition method least affecting TPB properties (in particular it is hygroscopic). In the same way photomultiplier behaviour at LAr temperature was heavily tested both by the supplier and the collaboration.

Light and charge transport in LAr is a delicate matter as well. Substances like $\mathrm{O}_{2}, \mathrm{H}_{2} \mathrm{O}, \mathrm{CO}_{2}$, and $\mathrm{N}_{2}$ are commonly found even in pure 6.0 commercial Argon, as well as in most building materials (mainly plastics). The first three substances are electronegative, so that they can attach drifting ionization electrons, thus quenching the charge yield. On the other hand nitrogen and oxygen can also produce light losses, by nonradiative interactions with Argon dimers before their decay. The WArP Collaboration has carried on dedicated studies on the effects of $\mathrm{N}_{2}$ [29] and $\mathrm{O}_{2}$ [30] contamination in liquid Argon, as a part of its R\&D program. The dependence 


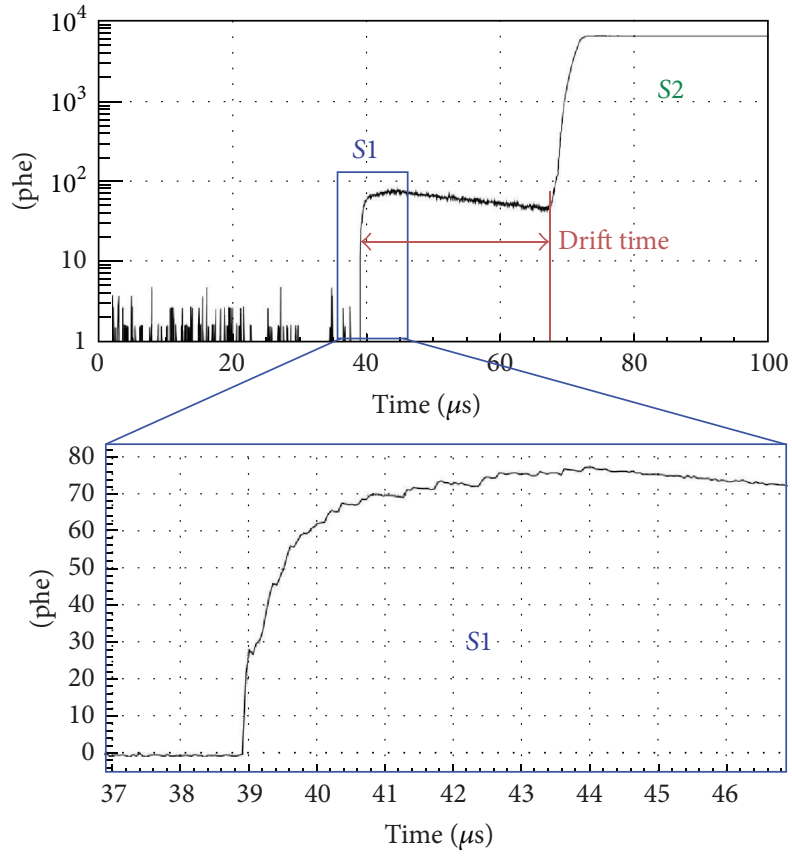

(a) Electron

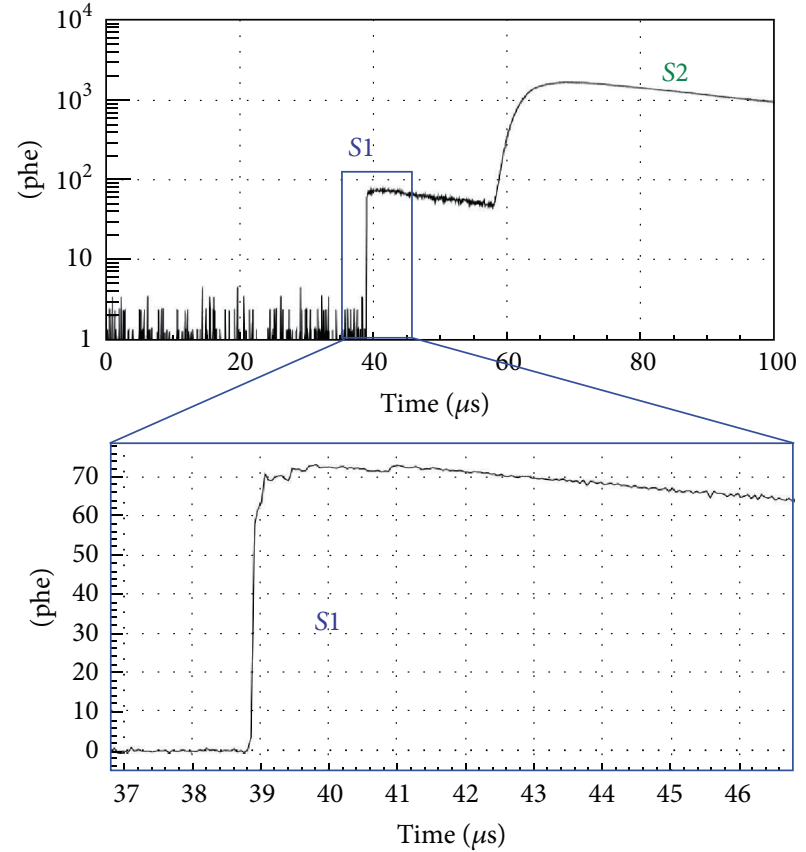

(b) Argon recoil

FIGURE 2: Example of collected integrated signals, due to electrons (a) and nuclear recoils (b). The difference in the magnitude of the secondary signal $s 2$ and in the rise time of the primary pulse (related to the fraction of fast light component $s f$ ) is clearly visible for the two classes of events.

of the long-lived light decay-constant and of electron life time on impurity concentration was demonstrated [30]. However, this effect sets in from values of the order of $0.1-$ $1 \mathrm{ppm}$, which are far higher than the one needed to correctly operate a detector (order of $\mathrm{ppb}$ for $\mathrm{O}_{2}$; see discussion below about impurity concentration control). $\mathrm{O}_{2}$ has a far higher interaction rate and affects both light and charge, but it can be extracted from LAr with dedicated filters; on the other hand, $\mathrm{N}_{2}$ affects only light, but it cannot be removed. To study the effects of $\mathrm{N}_{2}$ and $\mathrm{O}_{2}$, two chambers were employed, the 2.31 prototype and a 0.71 dedicated cell, where it was possible to inject predetermined amounts of impurities.

In an actual detector, impurity concentration has to be kept under strict control: highly purified commercial Argon has an impurity concentration below $0.1 \mathrm{ppm} \mathrm{O}_{2}$ equivalent, which can still affect both light and charge yield. Standard operating procedure to further lower the impurity levels is to implement a liquid and gaseous recirculation system where Argon is periodically extracted from the main volume and passed to a combination of purifiers. The solution devised for the ICARUS detector [13] implies the use of standard commercial filters such as Hydrosorb and Oxysorb. The first is a mechanical porous filter used to stop large molecules like $\mathrm{H}_{2} \mathrm{O}$ and $\mathrm{CO}_{2}$, while the second is a chemical filter used to stop small nonpolar substances like $\mathrm{O}_{2}$. In the WArP experiment a variation is introduced by employing Hopkalite rather than Oxysorb, as it is known to introduce less radiochemical contaminants. The combined use of the filters can reduce the overall impurity concentration up to a factor 1000. However, they are not sufficient to maintain purity over long time periods. As a matter of fact further contaminants can enter the sensitive volume, either from possible small leaks from the outside or from outgassing of the inner walls. The main impurities introduced in such way are water and oxygen; the effect is more important in the gas phase, as in liquid the molecules can freeze out on the wall surface and practically not diffuse into the sensitive volume. For this reason, in order to maintain a high level of Argon purity over long periods of time, a recirculation system is needed. The purity of the system then results from the balance between the inflow (leaks, outgassing) and the outflow (recirculation, filters) of impurities in the sensitive volume.

\section{The WArP Experiment}

The Wimp Argon Programme, as already mentioned in Section 2, started in the 1990s from ICARUS studies on the possibility to use scintillation light in noble liquids to perform separation between $\beta / \gamma$-induced events and nuclear recoils ([13-15]). Following and complementing the R\&D activities discussed in the previous section, in 2000 the assembly of a 2.31 demonstrator chamber was started, with the aim of testing and characterising the detection technique [22]. This chamber has been used widely so far, also to provide the first results on WIMP parameters within the WArP project [28], and later to test new materials and devices for further steps of the program [31]. Other than that, smaller chambers (order of $0.5 \div 11 \mathrm{LAr}$ ) were built, to carry on dedicated tests on new materials and photomultipliers [31]. Finally in 


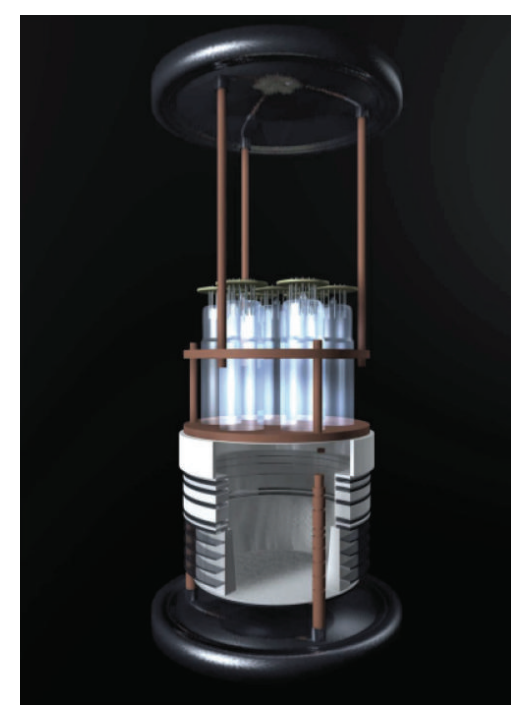

Figure 3: 3D layout of the 2.31 prototype, configuration for LNGS physics run. Details in text.

2005 there was the start of activities related to a full scale, 100 litres physics detector, aimed at performing a physics run and upgradable to larger volumes in case of program success. The detector was constructed and commissioned, but the mentioned problems in operation finally redirected the project scope to a technical run, useful to characterise the detection technology at the level of a full-scale detector.

3.1. 2.31 Prototype Results. The 2.31 chamber $[22,28]$ was first developed and tested with a single $8^{\prime \prime}$ photomultiplier (Electron Tubes 9357FLA), at sea level in Pavia, Italy. The chamber was immersed in a LAr bath, and it was structured as a Teflon cylinder surmounting an inverted truncated cone; all inner surfaces were covered by a layer of VM2000 reflector, with TPB deposited on it. The chamber contained a liquid volume capped by a gas pocket; the drift field in the liquid was set at $1 \mathrm{kV} / \mathrm{cm}$, while a system of 3 grids around the liquidgas interface assured electron extraction and multiplication. Geometry and dimensions details are reported in $[22,28]$.

The chamber was mainly used to evaluate the light collection efficiency and to test the production of secondary signals. It first demonstrated that different events can be reliably discriminated on the base of their $s 2 / s 1$ ratio: indeed low-ionising particles (e.g., electrons) produce small ionization density, and a lot of ionization electrons are extracted from the original interaction region, thus not recombining. Then a large secondary signal is produced. On the other hand, $\alpha$ 's and recoiling Argon nuclei produce high ionization density that enhances recombination, independently of the field strength. Therefore, few electrons are extracted and the resulting secondary signal is reduced.

In the same way it was shown that the shape of primary signals is different according to the particle nature: this is due to the ratio between the fast and slow scintillation light intensity, which depends on the interacting particle, as discussed in Section 2. The measurements led to evaluate a probability of wrong particle recognition of $10^{-4}$ for each investigated technique $(s 2 / s 1$ and pulse shape discrimination). As the two rejection techniques are independent and evaluated separately, an overall $10^{-8}$ rejection power can be estimated [22].

Later, the chamber configuration was modified, and the $8^{\prime \prime}$ PMT was replaced with seven $2^{\prime \prime}$ photo-tubes, ETL D757-UFLA (such implementation is shown in Figure 3). It was transferred at LNGS in 2005 to operate underground [28]. There it was first employed to study backgrounds in the underground laboratory, with particular attention to the ${ }^{39} \mathrm{Ar}$ spectrum and its specific activity. The light yield of the chamber for nuclear recoil events was evaluated, with a field of $1 \mathrm{kV} / \mathrm{cm}$. This was done by studying the recoil energy spectrum of neutron interactions, both from environmental radiation and from a dedicated Am-Be source. The collected data were compared with a GEANT4-based Monte Carlo simulation: as a result in both configurations the experimental spectrum could be reproduced by a constant light yield [28],

$$
\begin{gathered}
L Y_{\mathrm{env}} \approx 1.55 \pm 0.40 \mathrm{phe} / \mathrm{keV} \\
L Y_{\mathrm{AmBe}}=1.26 \pm 0.15 \mathrm{phe} / \mathrm{keV}
\end{gathered}
$$

The errors on the data are mainly systematic, deriving from uncertainties in the determination of the MC neutron flux induced inside the chamber.

The study of the proportional signal was again performed with events induced by electrons, $\alpha$ 's from ${ }^{222} \mathrm{Rn}$ and nuclear recoils. Electron-induced events result in a large ionization signal, while $\alpha$ 's produce a much lower one, as discussed above. Nuclear recoils s2/s1-ratio instead is found to depend on the recoil energy, and it is modelled by an empirical $(a+$ $b / E_{R}$ ) function, related to the recoil energy spectrum of the used source [22]. At high energies the ratio tends to the $\alpha$ particles value.

The activities on the 2.31 chamber ended with an underground physics run at LNGS, during which $2.8 \cdot 10^{7}$ triggers were collected, with a total exposure of $96.5 \mathrm{~kg} \mathrm{~d}$. By using the information from $\mathrm{s} 2 / \mathrm{s} 1$ ratio and the pulse shape analysis, nuclear recoil events were searched for. In the energy region below $55 \mathrm{keV}, 8$ events survived cuts, while nothing was detected at higher energies. The nature of these events was not understood: they could be of spurious origin, namely, residual neutrons or misrecognized e-like events, surviving cuts due to some inefficiency in the rejection. $90 \%$ C.L. upper limits were set on the WIMP $(m, \sigma)$ parameter space, for the case of no events observed at $E>55 \mathrm{keV}$. In the plot shown in Figure 4, WArP limits are reported, along with contemporary CRESST [32], EDELWEISS [33], and CDMS [34-36] ones. Though present limits are 3 orders of magnitude better [6-8], it can be seen how the demonstrator limits were comparable to those of contemporary, full-scale experiments. The allowed region for DAMA [9] was excluded as well.

The collected data were extrapolated to the upcoming 1001 detector, by correcting for the PMT quantum efficiencies, different photocathodic coverage, and dimensions; 


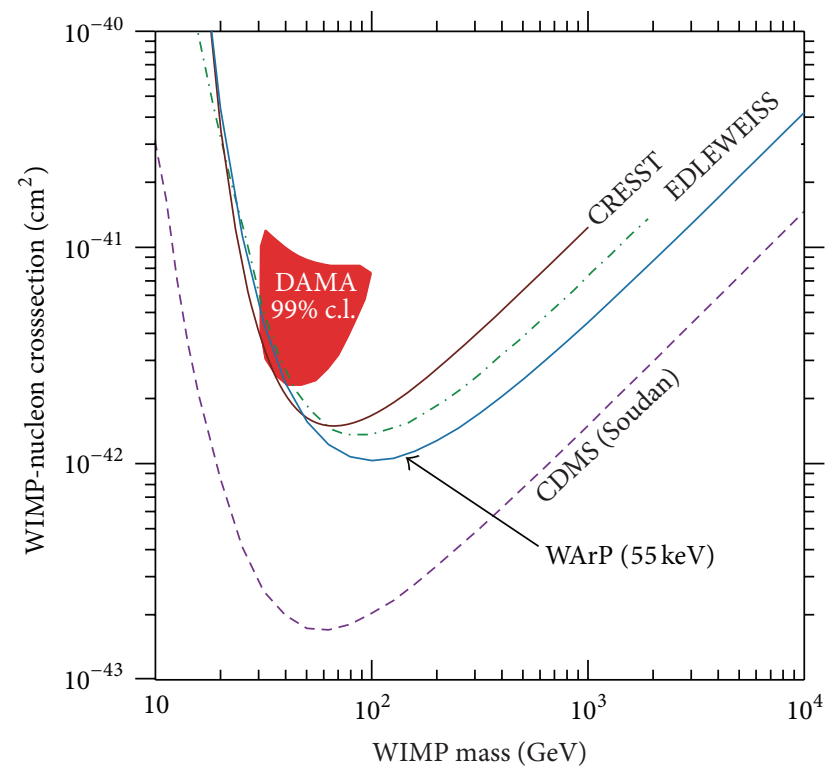

Figure 4: 90\% C.L. spin-independent limit for WArP-2.31 (solid blue line), compared with previous limits [28] available at the time.

as a result, a light yield value of $\approx 3.5 \mathrm{phe} / \mathrm{keV}$ in the inner detector (no field) was expected. It was estimated that the new detector sensitivity would have allowed to probe the region down to cross sections of the order of $10^{-45} \mathrm{~cm}^{2}$ in the mass region of $50-100 \mathrm{GeV}$, that is, the same order of magnitude of the limits reached today by Xenon100 $[6,7]$.

\subsection{WArP 100 l Detector. The 1001 detector is the last iteration} of the project: it consists of an inner double-phase chamber, which is surrounded by a larger volume of LAr, instrumented with photomultipliers as well. This serves as anticoincidence to reject all those events producing a signal in both volumes, and it is a unique feature first introduced by the WArP programme, to effectively eliminate spurious nuclear-recoil signals, produced by neutron elastic scatterings. The detector has been housed underground in Hall B of the Gran Sasso laboratories, in order to minimise its exposure to cosmic and environmental radiation.

The inner detector is a double-phase chamber, with a volume of 1001 (140 kg) of Liquid Argon topped by a gas pocket, read by 37 PMTs (Figure 5(a)). The drift region is delimited at the bottom by an Oxygen-Free Copper cathode ( $3 \mathrm{~mm}$ thick, $580 \mathrm{~mm}$ wide); the side walls are constituted by Oxygen-Free Copper field-shaping strips (race-tracks, $1 \mathrm{~cm}$ wide, $1 \mathrm{~cm}$ pitched), printed on a Kapton substrate. The system ensures a uniform field within the drift region; the strips are arranged in a truncated conical shape, to avoid trapping of ionization electrons between race-tracks themselves. The drift volume is closed at the top by the first of a three-grid system. Each grid is made by a stainless steel ring $(5 \mathrm{~mm}$ thick, $580 \mathrm{~mm}$ external diameter, $500 \mathrm{~mm}$ internal) holding stainless steel wires ( $4 \mathrm{~mm}$ pitch, $150 \mu \mathrm{m}$ diameter) enrolled on gold-plated bushes and mechanically tensioned to $750 \mathrm{~g}$. They are supported by $P E E K$ annular insulators, connected to the main structure. The described design defines a maximum drift length of $600 \mathrm{~mm}$. A resistive divider chain distributes supply voltage from the cathode, through the race-track system, to the first grid. The second and third grids are independently fed, which allows to (i) tune the field in the extraction/multiplication region, (ii) maximize the transparency of first and second grids, and (iii) achieve complete charge collection on the third one. The plane of the light-collecting photomultipliers is positioned $25 \mathrm{~mm}$ above the third grid: they are kept in place by the last layer of the PEEK structure that also sustains the race tracks and the grids. The PMTs and the gas pocket are enclosed in a stainless steel cap, vacuum insulated and fixed on the PEEK structure. A set of small resistors is placed just below the liquid surface: they assure continuous boiling of the liquid. Excess gas is evacuated through small holes in the cap, positioned at the desired level of the liquid-vapour interface ( $5 \mathrm{~mm}$ above the first grid), which is thus precisely set. The 37 PMTs, (31, 3 " ETL D750-UKFLA and 6, 2" ETL D757UFLA) are deployed in a hexagonal shape. This arrangement is chosen to maximise the photocathodic coverage, to a value of $10 \%$. The internal walls of the detector are covered with TPB evaporated on VM2000, which ensures conversion of VUV photons into visible light and minimises light losses. PMT windows are also treated with TPB. The whole inner detector structure is enclosed by a foil of copper, put to ground to isolate the internal volume and ensure that no field is present in the outer veto region.

The inner detector is immersed in a liquid Argon bath and surrounded by the active shield or veto (Figure 5(b)). This is a large ellipsoidal structure (5600 l volume) separating the internal detector from the cryostat and passive shield walls. It is made by a thin tile structure connected to a Copper superstructure. This is made to sustain the active shield weight during construction and its buoyancy after immersion in LAr. Each of the tiles is in copper, covered by a TPB+reflector layer; they have holes to house photomultipliers: in total 300 (36, $2^{\prime \prime}$ and $264,3^{\prime \prime}$ ) PMTs are in place in the veto, yielding a $7 \%$ photocathodic coverage.

The inner detector and the veto are contained in the main cryostat, made of AISI 304L stainless steel, chosen for its low radioactive content (for a detailed list of contaminants, see, e.g., [37]). The structure double walls are vacuum superinsulated. It is closed by a top flange from which the copper superstructure hangs, sustaining both the inner and veto volumes, which then make a single object (see Figure 5(b)). On the dewar inner walls, a $10 \mathrm{~cm}$ polyethylene shield is mounted, used to moderate the neutrons produced by the radio-contaminants present in the cryostat walls. The dewar inner volume is $30 \mathrm{~m}^{3}$, of which 23 are filled with LAr.

The cryostat is surrounded by an external shielding system, to protect the detector from $\gamma$ and neutron radiation, as well as to serve as further container, in case of significant cryostat spills. It is made by a $10 \mathrm{~cm}$ thick lead layer, contained in stainless steel boxes, followed by $70 \mathrm{~cm}$ of polyethylene. The shield was not completed during the actual technical run of the detector. An external antiseismic structure, made of carbon steel, sustains the external shielding. 


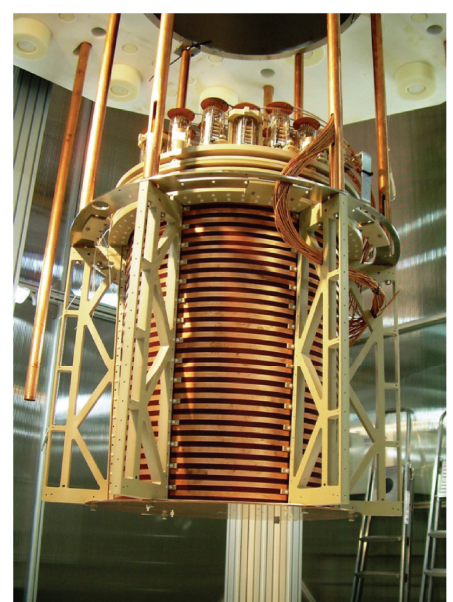

(a)

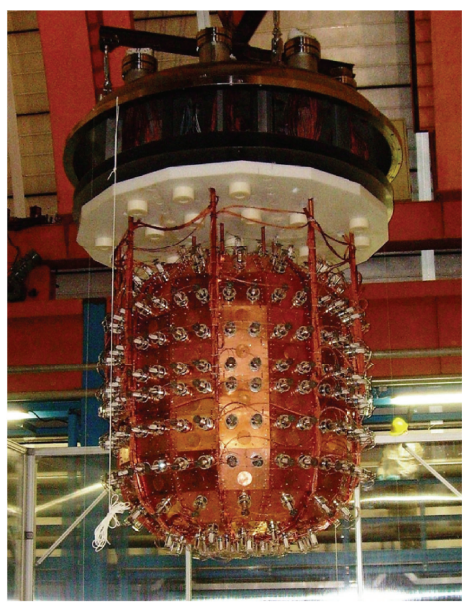

(b)

Figure 5: (a) Picture of the inner detector, completed in the clean room. (b) Completed WArP 1001 detector, ready to be inserted in its cryostat. The veto is visible, hanging from the cryostat top flange; the inner detector is inside.

The cryogenic system of the experiment consists in the main dewar, the filters for Argon purification, and the filling/recirculation units. The filling unit is used during data taking for cryostat periodical refills from an external storage dewar, connected to the main one through a Hydrosorb-Hopkalite cartridge immersed in a LAr bath. During 2011 run, the consumption of Argon was measured in $900 \mathrm{l} /$ day. The gas recirculation unit is used to counter the effect of outgassing: once extracted from the detector top, gaseous Argon is then made to pass through three standard filters in parallel and then recondensed and injected at the bottom of the cryostat. Nominal recirculation speed is $5 \mathrm{l} / \mathrm{h}$. A further pump for liquid recirculation was later mounted, but it was only briefly used during 2011 data taking, normally keeping only the gas recirculation operational.

The inner and veto photomultipliers are managed by two different types of electronics: the signals from the veto PMTs are sent to 40, 8-channel, N914 CAEN boards. The analog sums of the signals are then sent to 5 CAEN V1724 digitisers, with sampling frequency of $100 \mathrm{MHz}$ and resolution of 14 bits. The signals of the 37 inner detector photomultipliers are sent to 19 Aquiris Boards, DP235 Dual-Channel PCI Digitizer Card, with a sampling up to $1 \mathrm{GS} / \mathrm{s}$ and 8 bit dynamic range.

\subsubsection{Trigger Configuration. The data acquisition (DAQ)} system of the WArP detector is based on a double-level trigger specific of the inner volume that can be coupled to the trigger of the veto. The two systems are completely independent and can be operated separately to study the performance of the two devices. The overall system is managed by a dedicated LAbView interface.

In principle the veto should operate in conjunction with the inner detector; however, when operated alone for tests, its trigger system is made to start the acquisition when the sum of the integrated signals from all the PMTs overcomes an overall threshold of 100 phe, set a priori.
The inner detector first-level trigger is a simple lower threshold on the signal height, which must be overcome by a minimum number of PMTs in coincidence, in order for the signal to be registered. The minimum number of coincident photomultipliers (multiplicity), as well as the threshold value, had to be set in order to effectively reject noise, without losing too many real signals. After test runs, the multiplicity was set to four and the threshold to $15 \mathrm{ADC}$ counts (ADCc).

The second-level trigger consists in a live integration of the signal, starting with a short delay (100 ns) after its onset. Electron-induced pulses, dominated by the slow light component, are mostly integrated, while fast, recoil signals are almost completely skipped. By setting an upper threshold for the integrated pulses not to overcome, slow events are rejected, whereas fast events are accepted.

True rejection efficiency achieved with this procedure has yet to be evaluated, as it was not regularly implemented during data taking. Instead, only the first-level trigger was used, in order to collect electron-recoil data, mainly from ${ }^{39} \mathrm{Ar}$ decay, to test the detector response. A few dedicated runs were performed with the second-level trigger enabled, during which the acquisition rate was significantly lowered from $30-40 \mathrm{~Hz}$ to $12 \mathrm{~Hz}$. Visual inspection of the collected data shows a significant depression of the ${ }^{39}$ Ar spectrum, but not its complete disappearance. While quantitative analyses on this on-line rejection power have yet to be performed, the comparison of spectra already confirms the possibility of achieving at least partial background rejection online.

3.2.2. Detector History. The activities on WArP 1001 [38] started in 2005 when, prior to the detector construction, all the building materials were selected and tested for low radioactivity requirements. The inner detector and the veto were preassembled in Pavia laboratory, to test and qualify assembly procedures and mechanical tolerances. Later, the detector was disassembled and the components underwent special cleaning by an external industry before 
being sent to LNGS. Meanwhile photomultipliers were heavily tested, both at room and $\mathrm{LN}_{2}$ temperature. Manufacturer tests were followed by additional custom measurements. The tests regarded stability at room and $\mathrm{LN}_{2}$ temperature, intrinsic noise evaluation, and compliance to specifications.

Main assembly started in LNGS Hall B in 2007: the external structures and the cryostat were mounted, while the detector was assembled in a dedicated clean room. In parallel PMT preassembly was carried on, as well as TPB evaporation on the inner surfaces. Final assembly took place in the clean room from June to December 2008. After all the PMTs had been tested in dark conditions, the detector was moved into the cryostat. Figure 5(b) shows the assembled detector ready to be inserted into the cryostat. The external cooling and purification systems were first installed in January 2009. At the same time the external lead shield walls were put in place; the installation of the lateral polyethylene external shield was started as well, but it was not completed.

The WArP 1001 detector experienced some technical issues, mainly related to HV distribution, which severely hindered operation in double-phase mode with a stable electric field in the liquid. The nominal operating drift field should have been of $1 \mathrm{kV} / \mathrm{cm}$. The detector first started commissioning in 2009, right after assembly was concluded, but a short circuit problem on the supply chain prevented its operation. Similarly, in 2010 detector was commissioned, but another HV failure again stopped its operation, after only two days of activity. Before attempting a novel start, some elements underwent further checks, due to detected instabilities on the photomultipliers: the elements of the PMT bases (resistors, capacitors) were again tested for stability at low temperature, and the veto PMTs were optically decoupled with Kapton foils.

After that, a third commissioning was started in 2011. All the internal volumes were evacuated starting in early April 2011. Vacuum pumping lasted about two months: once target pressure less than $10^{-4}$ mbar had been reached, pumping continued to ensure outgassing on the internal surfaces. During this phase, repeated washings with gaseous Argon were performed, to further lower water contaminations.

Vacuum pumping was stopped on May 25th, with a measured internal pressure of $1.4 \times 10^{-5}$ mbar. Cooling phase then began, with the use of a liquid Argon serpentine, installed between the internal and external walls of the main cryostat. At the end of this phase, the measured residual pressure of water was around $3 \times 10^{-9}$ mbar, while oxygen and nitrogen concentrations were, respectively, 10 and 100 times higher.

Within five days, below-zero temperature was reached uniformly inside the cryostat, and the filling phase began. Initial filling was performed during a two-day period, May 30th to June 1st, with 6.0 commercial liquid Argon. The load liquid was further purified before injection, by passing through an external Hopkalite filter immersed in a LAr bath. Initially the liquid was used to further cool down the inner volume; one of the Argon lines was left open to vent the produced excess gas; after this first phase, filling proceeded at a speed of $500 \mathrm{l} / \mathrm{h}$. This last stage ended after the inner polyethylene shield had been submerged. Right after completion, the GAr recirculation unit was turned on; later also the liquid recirculation pump was put into operation. Finally the voltage supply for the veto and inner detector photomultipliers was turned on, successfully ending commissioning.

Data acquisition took place from July 24th to November 21st. The first tests on HV supply were carried on one week after filling, down to $-45 \mathrm{kV}$ without problems (i.e., discharges). At the beginning of July, the official raise of the field was started. Foreseen to reach $-60 \mathrm{kV}$ in two days, the procedure was stopped at $-20 \mathrm{kV}$, when an instability occurred, followed by an anomalous current flow. This prevented the achievement of nominal field conditions at $1 \mathrm{kV} / \mathrm{cm}$. Despite this problem, data taking with electric field began, starting with $-10 \mathrm{kV}$ supplies.

Later, HV supply could be slowly raised, and the highest stable field reached during data taking was $270 \mathrm{~V} / \mathrm{cm}$, that is, a voltage of $-25 \mathrm{kV}$. Runs were also taken at $-27,-30 \mathrm{kV}$, but discharges were usually experienced. The system seemed to deteriorate under continued stress, as back-to-back runs caused higher rate of discharges with time. Acquiring data in zero-field conditions for a time led to more stable conditions, allowing running steadily without further discharges. After the decommissioning, the inner detector was extracted and opened: a deterioration of the insulation of the HV cable was found, as well as a temperature-probe cable disconnected and floating free in the liquid inner volume. The HV problems led to the early end of the 2011 run and to ultimate closure of the WArP programme.

\section{2011 Data Analysis}

During the 2011 campaign 151 runs were acquired in zerofield conditions. Of them, 121 are in "standard mode," that is, with a $15 \mathrm{ADCc}$ threshold for the signal registered by the single PMTs and with acquisition triggered by coincident firing (multiplicity) of 4 units. The remaining 30 runs are not considered here, as they were expressly used to test different thresholds, multiplicities, and the second level trigger. 13.718.000 total events were collected.

On the other hand, 66 runs were taken in nonzero field conditions, for a total of 1.059 .000 events, although in different configurations. The second and third grids ( $G 2$ and G3), independently fed, were always kept at $V(G 2)=$ $+900 \mathrm{~V}, V(G 3)=-700 \mathrm{~V}$, to provide, respectively, electron extraction/multiplication and then collection on G3. HV supply was brought from -10 to $-30 \mathrm{kV}$, for a corresponding drift field ranging from 90 to $330 \mathrm{~V} / \mathrm{cm}$. Electron drift velocity in these field conditions lies in the range $0.499 \div 1.297 \mathrm{~mm} / \mu \mathrm{s}$ $[39,40]$.

4.1. Background Sources. The 1001 detector has been described by Monte Carlo simulations, both with FluKa [41] and GEANT4 [42] packages, before its actual construction $[22,27]$. The main goal of these simulations was to evaluate 
the main sources of background expected during data taking and then to estimate the minimum rejection power needed to eliminate the spurious signals.

Two main sources of background are present:

(i) electron recoils produced by $\beta / \gamma$ emitters, which may be misrecognized as nuclear recoils;

(ii) nuclear recoils produced by elastic scattering of neutral particles like neutrons and, possibly, neutrinos.

As regards electrons, the main elements to be considered are Argon isotopes $\left({ }^{39} \mathrm{Ar},{ }^{41} \mathrm{Ar},{ }^{42} \mathrm{Ar}\right),{ }^{85} \mathrm{Kr}$, and ${ }^{42} \mathrm{~K}$, all $\beta^{-}$decaying. Calculations show that all contributions are negligible with respect to ${ }^{39} \mathrm{Ar}$, which produces, in the acceptance energy window of the experiment, some $10^{7}$ events in 100 days of acquisition. To be able to exclude these events, it is necessary to apply the combined analysis on pulse shape and $s 2 / s 1$ ratio. Electron events can also be produced by $\gamma$-emitters from the $\mathrm{U}, \mathrm{Th}, \mathrm{K}$ chains, trapped in the building materials. While such events can be rejected by exploiting both pulse shape discrimination and $s 2 / s 1$ ratio, as for $\beta^{-}$decays, further means are available in this case: it is expected that low energy $\gamma^{\prime}$ 's $(E<30 \mathrm{keV})$ would interact via photoelectric effect near the detector walls (where they are produced). Then volume cuts should allow to get rid of such events. On the other hand, $\gamma$ 's with higher energy $(E>30 \mathrm{keV})$ could produce multiple Compton scatterings: in this case rejection can be achieved by resolution of multiple events.

Neutrons sources are varied: they are produced in fission or $(\alpha, n)$ reactions of the radioactive contaminants in the building/surrounding materials, or following cosmic rays interactions. Moreover, a component from environmental radioactivity is expected: the measured neutron flux (for energies above $1 \mathrm{MeV}$ ) in LNGS [43] is $6 \times 10^{-7} \mathrm{~cm}^{-2} \mathrm{~s}^{-1}$; the external polyethylene shield is expected from simulations to reduce this flux by a factor $5 \times 10^{4}$. Neutron-induced nuclear recoils mimic WIMP interactions; however, they are likely to produce multiple interactions, due to their much higher cross section. Simulations were used to evaluate the amount of neutron events that cannot be rejected, according to the radioactive content of the building materials and as a function of the signal thresholds used for the veto and inner detector. The best way to reject these events is to detect double interactions, either in the inner volume alone or both there and in the veto. Thus, the active shield threshold should be in principle as low as possible. The expected values of light yield for the $100 \mathrm{l}$ detector, extrapolated before construction from the 2.31 data in zero-field conditions, were 3.5 (3.0) phe/ $\mathrm{keV}$ in the inner detector (veto) for electron events and $\approx 0.65 \mathrm{phe} / \mathrm{keV}$ in both volumes for nuclear recoils. With these values and assuming thresholds of $30 \mathrm{keV}$ in the two detectors, simulations reported that the main source of background neutrons came from inner photomultipliers; with the help of veto and resolution of multiple interactions, the number of residual unvetoed events was estimated to be around 3/year.

Finally, neutrino-nucleus elastic scatterings (from solar and cosmic-ray neutrinos) can induce recoil events in the same energy region selected for WIMPs. Given the weak cross sections involved in this case, passive/active shielding is of no use and this background is irreducible. However, the cosmic neutrino flux at LNGS is estimated in $\approx 11.5 \mathrm{~cm}^{-2} \mathrm{~s}^{-1}$ [44], yielding an event rate in the energy range of interest of the order of $10^{-6}$ event/day. Such a rate is negligible over a datataking period of a few years.

In the original WArP proposal, the goal for background rejection was set at a limit of 1 event in 100 days wrongly recognised. The tests on the 2.31 chamber $[22,28]$ show that at least it is possible to achieve a $10^{-4}$ rejection power for electron events for each of the two, independent analysis techniques. Given that the strongest source of electron background events was evaluated in some $10^{7}$ event in 100 days within the detector acceptance window, as mentioned, the evaluated combined rejection power should allow reaching the requested goal.

The operation of the 1001 detector in 2011 showed lower values of light yield (discussed in the next sections); then background evaluations need to be rerun to correctly fit the new acceptance windows of the detector, in terms of collected photoelectrons. This has been possible thanks to a new, recently developed simulation [37], able to reproduce the light yield data of the 2.31 prototype and of a $0.7 \mathrm{~kg}$ chamber used for PMT testing. The code has been validated on both chambers and it can now be applied to the 1001 detector with two main objectives: (i) rerun, as said, the background evaluation, possibly in parallel with older codes to check results and (ii) study the effect of growing detector dimensions on light loss and collection (i.e., variations in light yield, given the same used materials and photocathode coverage).

A further source of background is related to $\alpha$-emission. This is mainly due to ${ }^{222} \mathrm{Rn}$ which, with a half-life of $3.82 \mathrm{~d}$, should rapidly disappear from the data (as demonstrated in [22] for the 2.31 prototype). However, in the 2011 campaign a significant presence of such events was steadily recorded, meaning a source of Radon was present inside the detector, continuously contaminating it. The presence of Radon is a very dangerous background for the experiment, as $\alpha$ particle signals share similarities with nuclear recoils (mostly regarding the primary pulse shape). Unfortunately, so far it has not been possible to identify the source of Radon contamination inside the detector and act to remove it.

4.2. Data Code. The data analysis code for the 1001 detector has been developed on the 2.31 prototype data. It basically operates in two steps: first, for each run the initial 5000 collected triggers are used to produce the single electron response (SER) of each photomultiplier that is then saved in a database. This represents the response of the PMT to single impinging photons and it is used to calibrate the instrument [45].

Later, single triggers are analysed: the start of primary pulse on each PMT waveform is searched for and then checks for signal saturation are performed. Baseline and noise information (RMS and peak-to-peak) is calculated in the pretrigger window ( $2 \mu$ s wide). The waveform is then reversed 
(positive edge) and the baseline is set to zero. To obtain the primary pulse amplitude, an integration of the waveform over $7 \mu$ s after trigger is done, while the fast component is set as the fraction of signal integrated in the first $80 \mathrm{~ns}$ after onset. If the primary signal is not saturated, then a secondary pulse is searched for and reconstructed as well. This is done for each PMT; then the signal intensity is normalized to the respective SER, to be expressed in phe. Finally, the summed up signals $(s 1$ and $s 2)$ are calculated. An example of summed signals is shown in Figure 6 for the different cases of electron-driven events and $\alpha$-particles ones.

Ancillary routines allow then to (i) visually inspect each single PMT waveform, to check reconstruction problems; (ii) perform more detailed analyses on single waveform, like Fast Fourier Transform (FFT) to study noise sources; (iii) create an average waveform for the run: according to the measurements performed on $\mathrm{N}_{2}$ contamination [29], the fitted value for the slow decay-time constant $\tau_{s}$ can be used to estimate the amount of light-quenching impurities.

The code was developed on 2.31 prototype data; then it had to be slightly adapted to the different conditions of the $100 \mathrm{l}$ detector. This was also due to the nonstandard conditions of operation: a far lower drift field than planned, meaning longer drift times, and an unidentified source of noise at $250 \mathrm{kHz}$ in the data. This only slightly affected primary reconstruction but could hinder reconstruction of long waveforms containing secondary pulse. The code has been modified during and after data taking (for details see [37]), and now it should be possible to recover field-on data affected by this noise superimposed on the waveforms.

4.3. Veto Data. The veto has been operated mainly independently during data taking. The response of the detector in terms of light yield has been evaluated through analysis of both electron recoils and $\alpha$-induced signals. Calibration to electron recoils was done with a ${ }^{60} \mathrm{Co}$ source inserted in the detector next to the inner structure: two gamma lines were detected (though not resolved) at 1.17 and $1.33 \mathrm{MeV}$. On the other hand, calibration to $\alpha$-particles was possible due to the presence of events from the ${ }^{222} \mathrm{Rn}$ chain: peaks from ${ }^{222} \mathrm{Rn}(5.59 \mathrm{MeV}),{ }^{218} \mathrm{Po}(6.11 \mathrm{MeV})$, and ${ }^{214} \mathrm{Po}(7.83 \mathrm{MeV})$ were identified in the background spectrum. The analysis of both sources, after correcting for the difference in response between $\alpha$-particles and electrons, led to a corresponding light yield evaluation of

$$
L Y_{\text {veto }} \sim 0.53 \mathrm{phe} / \mathrm{keV}
$$

This result is approximately 6 times smaller than extrapolations from the 2.31 prototype. The reason of this is yet to be fully understood, the main possibilities under investigation being a lower-than-evaluated quantum efficiency of the PMTs of the veto and/or an underestimated effect of the increasing detector dimensions on light survival. A new simulation of the 1001 detector, with the mentioned newer code used to reproduce the prototype chambers results, could possibly help verifying such hypotheses.
TABLE 2: Summary of light yield measurements from calibration lines (gamma sources) and background measurements, performed in November 2011. Errors from fits are negligible. Errors on LY for $\gamma$-sources are taken as the sigma of the photo-peak distribution.

\begin{tabular}{lcc}
\hline Nuclide & $\gamma$-Energy $(\mathrm{keV})$ & LY $(\mathrm{phe} / \mathrm{keV})$ \\
\hline${ }^{60} \mathrm{Co}$ & 1173 & $1.54 \pm 0.10$ \\
${ }^{60} \mathrm{Co}$ & 1335 & $1.57 \pm 0.06$ \\
${ }^{133} \mathrm{Ba}$ & 356 & $1.55 \pm 0.14$ \\
${ }^{137} \mathrm{Cs}$ & 662 & $1.54 \pm 0.09$ \\
\hline $\mathrm{Bkg}$ & Endpoint $(\mathrm{keV})$ & $\mathrm{LY}(\mathrm{phe} / \mathrm{keV})$ \\
\hline${ }^{39} \mathrm{Ar}$ & 565 & 1.58 \\
\hline
\end{tabular}

4.4. Inner PMTs Characterization. The data collected with the inner detector in zero-field conditions were firstly used to characterise the 37 photomultipliers and to inspect the system stability over time $[37,46]$. Data from 22 August to 21 November are considered, for a total of 92 live days. For each PMT three parameters are investigated, namely:

(i) the gain, derived from the SER peak information: it measures the number of charges collected at the anode of the PMT per photoelectron produced at the cathode [45];

(ii) the noise, root-mean-square (RMS) and peak-to-peak (PP), evaluated in the pretrigger window;

(iii) the counting rate, evaluated in the waveform region 8 to $13 \mu \mathrm{s}$ after the primary signal onset.

Five of the 37 PMTs were found to be nonworking at the start of data taking; therefore, in that case only the noise parameter was evaluated.

The gain data reveal an overall stable behaviour for most channels, centred on a value of $2 \times 10^{6} \mathrm{e}^{-} / \mathrm{ph}$. Few variations can be explained by changes in the DAQ configuration (e.g., reevaluation of some baselines). Only one channel shows significant instability, which can be ascribed to a malfunctioning of the PMT itself.

Noise values vary between the different channels, ranging from 0.15 to $0.3 \mathrm{mV}$ for RMS and from 1.1 to $2 \mathrm{mV}$ for peakto-peak. However, there is a remarkable correspondence between RMS and PP behaviors for each PMT, which ensures that no spurious peaks are wrongly labelled as good signals; moreover, very similar values are obtained for consecutive even-odd channels (e.g., 0-1, 2-3) which are read by the same acquisition board. This means that the detected noise can be related also to the boards themselves rather than only to the PMTs.

The rate data show a wide range of values, from $1-2 \mathrm{kHz}$ to $\approx 20 \mathrm{kHz}$. However, each PMT has a quite stable behaviour, only interrupted by a significant increase during a 15-day period, when the detector underwent a lot of stress, with repeated insertions of $\gamma$-calibration sources and several attempts to run in field-on conditions. After this period the rates lower again, demonstrating that the PMTs felt the changes but were able to recover later. Moreover, no corresponding decrease in gain was registered, which means that light collection system worked properly and the detector 


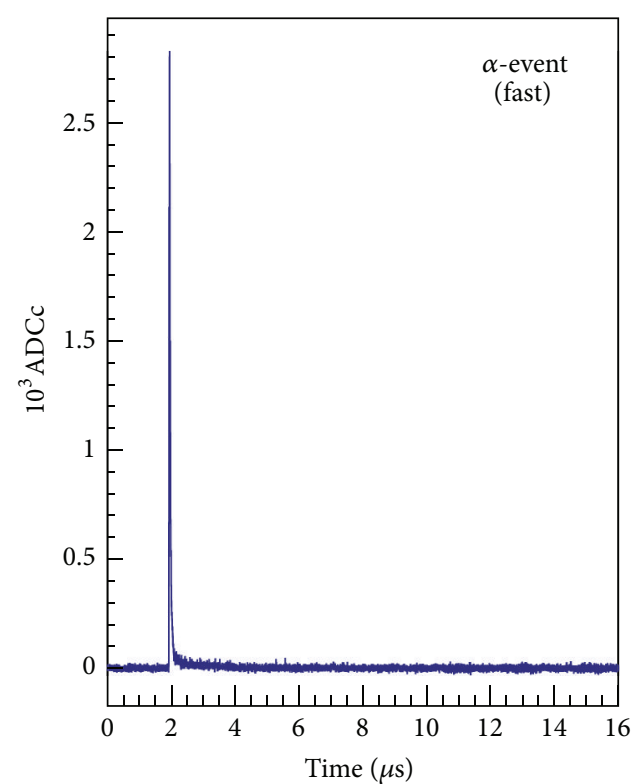

(a)

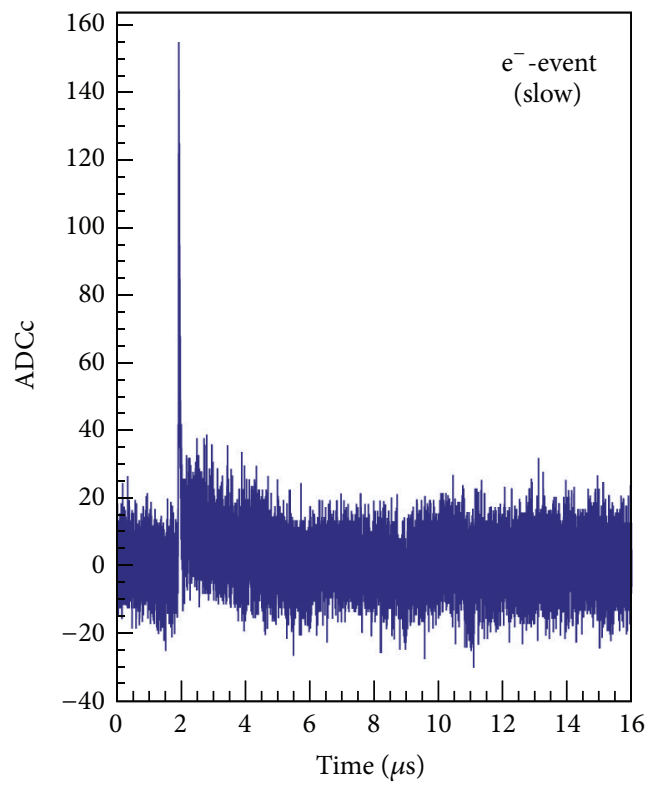

(c)

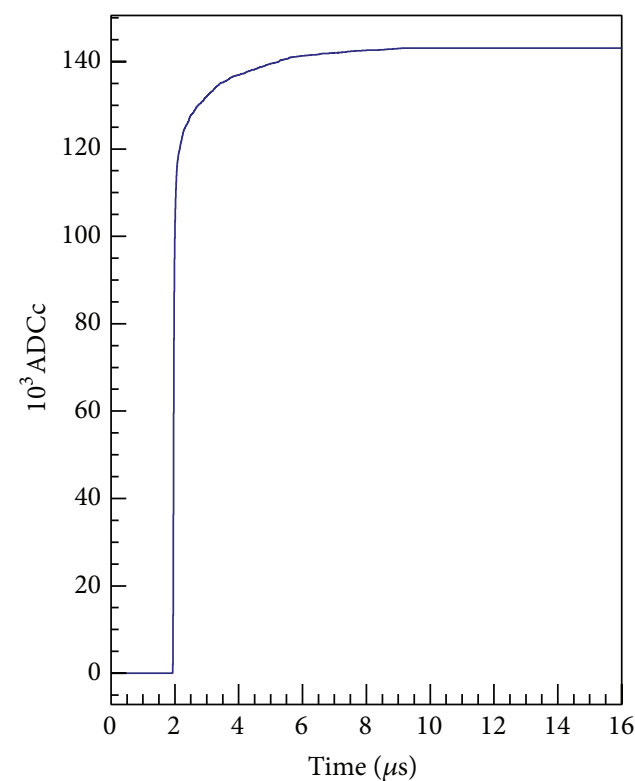

(b)

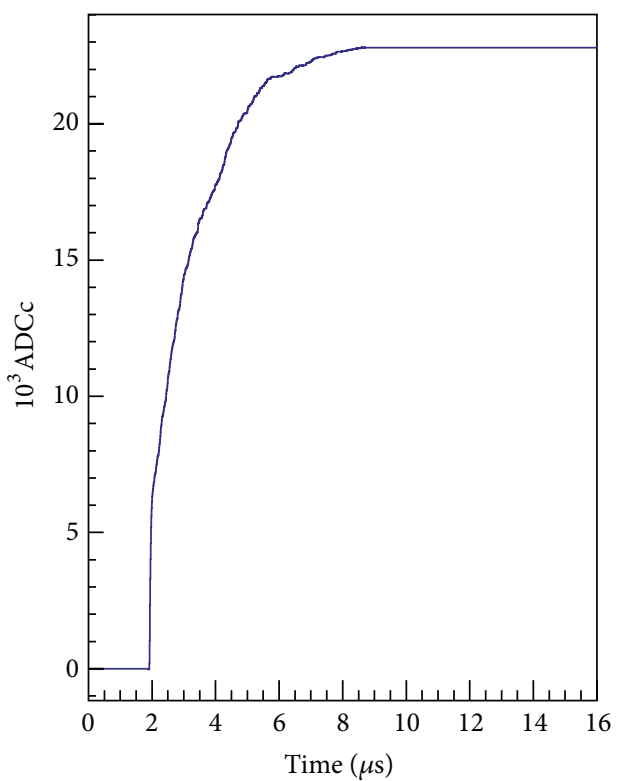

(d)

Figure 6: ((a), (b)) Total reconstructed waveform (sum of 37 PMTs, (a)) and integrated signal (b) for an $\alpha$-induced event in the inner detector. ((c), (d)) The same for an electron event. The difference in the relative weight of the fast and slow light components in the two classes of events is clearly visible in the rise times of the integrated signals, with the upper one being much smaller than the lower one (i.e., dominating fast component for the $\alpha$-particle event).

was able to recover from solicitations, allowing for a yearslong data taking period.

4.5. Inner Data, Zero Field. Runs in zero-field conditions were also used to study the detector response to different particles, in terms of light yield. Response to electronlike events was obtained by fitting the peaks of various $\gamma$ calibration sources and the $\beta$-spectrum of ${ }^{39} \mathrm{Ar}$, as shown in
Table 2 . An average value of $1.4 \div 1.6 \mathrm{phe} / \mathrm{keV}$ was measured over the whole acquisition period, slightly increasing with time. The value is compatible with the few measurements made on ${ }^{39}$ Ar 2010 data (1.6 phe/keV). The evolution of light yield was investigated in the same period chosen for PMT characterization. As mentioned, a slight increase is detected over time, which is ascribed to a stabilisation of the detector with time, despite the technical HV problems. A 15day period shows higher $L Y \sim 1.75 \mathrm{phe} / \mathrm{keV}$, corresponding to 


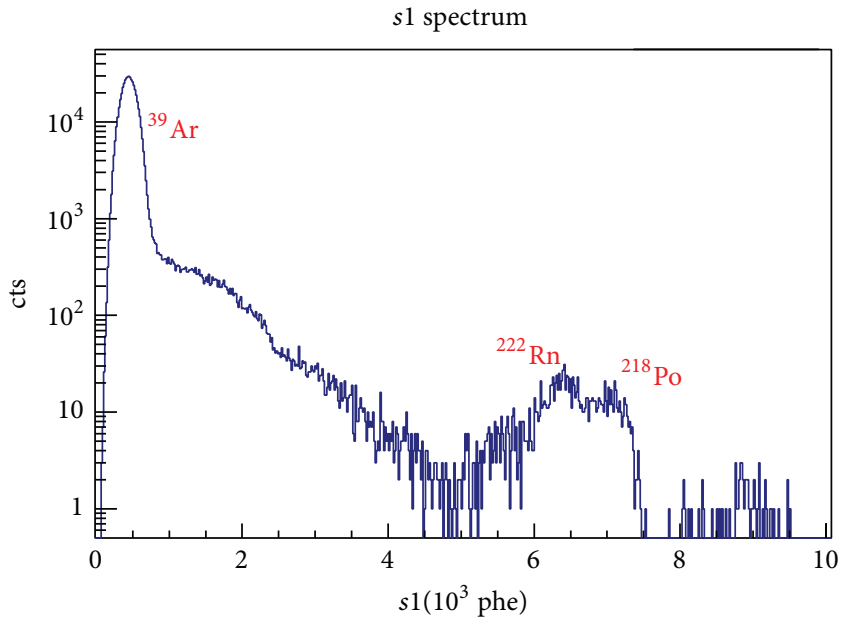

(a)

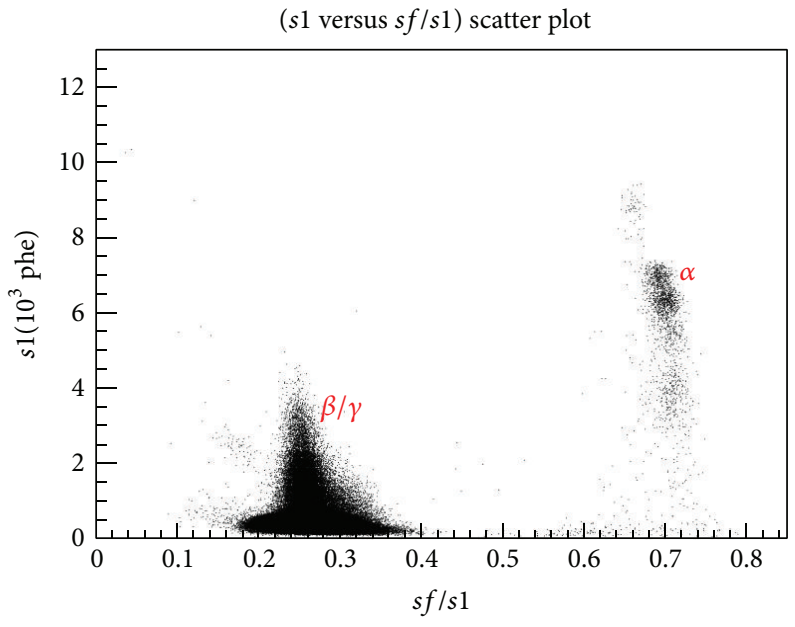

(b)

FIgURE 7: Primary signals collected with the WArP 1001 detector in 2011: (a) primary energy spectrum in units of phe. The low energy region is dominated by the ${ }^{39} \mathrm{Ar} \beta$-spectrum, then at higher energies signals from other radioactive contaminants are present. The $\alpha$-peaks of ${ }^{222} \mathrm{Rn}$ and ${ }^{218}$ Po are visible at $6-7 \times 10^{3}$ phe; (b) $(s 1$ versus $s f / s 1)$ scatter plot, showing the separation between slow events, induced by $\beta / \gamma$ radiation, and fast ones, mostly due to $\alpha$-decays.

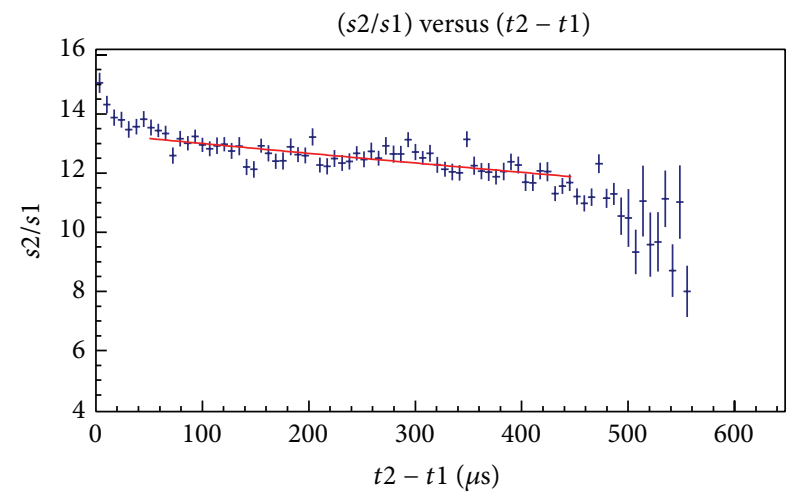

FIGURE 8: $s 2 / s 1$ ratio as a function of drift time $(t 2-t 1)$. The region used for an exponential fit (red line) allows estimating the electron life time. The decrease of the $s 2 / s 1$ value for the highest drift times is related to few badly reconstructed events near the cathode.

runs acquired with a higher single PMT threshold (25 ADCc instead of 15).

The reason of this could be due to the fact that, with a 15 ADCc threshold, noise rejection is not perfected, which could slightly lower the estimated response of the single PMTs and then the overall system response. However, the higher value of the threshold also increased the low-energy threshold of the recorded spectrum, cutting away most of the interesting window for WIMP interactions; therefore, the single PMT threshold was brought back to its original value, which represents a value of compromise.

In the data, the $\alpha$-peaks of the ${ }^{222} \mathrm{Rn}$ chain can be recognised, as in the veto (see Figure $7(\mathrm{a})$ ), but the signals are mostly saturated; therefore, no reliable evaluation of light yield could be performed in this case.
4.5.1. Pulse Shape Analysis. By studying the fraction of fast signal component per event versus the total pulse, it is possible to check the discrimination achieved for different classes of interacting particles: as seen in Figure 7(b), two distinct populations can be recognised. The one with $s f / s 1 \sim$ 0.3 represents events dominated by the slow scintillation light component; they are mainly due to ${ }^{39} \mathrm{Ar}$ decay and $\gamma$-background events. On the other hand, the population at $s f / s 1 \sim 0.7$ is instead due to fast events, and it can be roughly divided in three groups. The events with $>6000$ phe are mainly due to ${ }^{222} \mathrm{Rn}$ chain $\alpha$-decays. The region around $3000-4000$ phe shows a group of events not yet identified, but which could be due to low energy $\alpha$-decays of the Thorium chain. Finally in the region around 100 phe there is a group of events that could be due to background neutrons or $\alpha$ decay byproducts of contaminants in the detector walls. 3D localization is necessary to resolve the nature of this group. The recognition of a population of neutrons would be useful to test the detector to nuclear recoils, as there has not been, during the 2011 campaign, the possibility to insert neutron calibration sources.

4.6. Inner Data, Field-On. To verify the dependence on $\mathscr{E}_{\text {drift }}$ of the recombination process in Argon, the value of the light yield was measured with different field configurations, on ${ }^{39} \mathrm{Ar}$ spectra. A decrease was expected in the collected primary light (light yield), due to more ionization electrons being drifted away by the electric field. Indeed it was registered, at a field of $330 \mathrm{~V} / \mathrm{cm}$, a $32 \%$ reduction of primary light output, with respect to zero-field conditions. This is in agreement with previous studies on light/charge yield variations, as a function of the drift field intensity [47].

Field-on data can of course also be used to study the $s 2 / s 1$ ratio and the possibility to perform particle discrimination 
TABLE 3: Effective electron lifetime and signal attenuation (for particles travelling the whole volume) estimated for two-field values in WArP 1001.

\begin{tabular}{lcc}
\hline $\mathscr{E}_{\text {drift }}(\mathrm{V} / \mathrm{cm})$ & 210 & 330 \\
Drift time $t_{\text {drift }}(\mu \mathrm{s})$ & $544 \pm 2$ & $462 \pm 1$ \\
$\langle s 2 / s 1\rangle$ & 7 & 13 \\
Fitted $\tau_{e}(\mu \mathrm{s})$ & $\approx 1400$ & $\approx 3800$ \\
$e^{-}$signal attenuation & $33 \%$ & $12 \%$ \\
\hline
\end{tabular}

based on this. As mentioned above, the original analysis code was usually characterised by a bad reconstruction of secondary events. Modifications were carried on since then. Presently, only a small fraction of the available data has been correctly reanalyzed; however, preliminary analyses have been performed.

The behaviour of $s 2 / s 1$ as a function of drift time (indicated as $t 2-t 1=t_{\text {drift }}$ ) has been studied for electron recoils, and it is shown in Figure 8. Extremely pure Argon would result in a stable $s 2 / s 1$ ratio for signals from the whole chamber; on the other hand, a decrease of the ratio as a function of the distance from the liquid surface is the evidence of the presence of the mentioned electronegative impurities $\left(\mathrm{O}_{2}, \mathrm{H}_{2} \mathrm{O}\right)$ that can attach drifting electrons (see Section 2.1). The distance to the surface is directly proportional to the drift time through the electron drift velocity. In Table 3 the obtained average values of $s 2 / s 1$ are shown for electron recoils, along with estimates for the effective electron life time, $\tau_{e}$, and the corresponding signal attenuation within the chamber.

Electron life time is inversely proportional to impurity concentration, through a rate constant (see [30]) that is slightly decreasing with increasing field. However, here one should also take into account the effect of the issues in reconstruction of secondary signals: while an increase of $\tau_{e}$ with the field is expected, the difference between the reported values is also dependent on the mentioned dispersion of reconstructed data. This implies a significant uncertainty on the measurements, which should be taken as evaluations.

4.6.1. $3 D$ Localization. In principle, $3 \mathrm{D}$ localization can be performed on secondary signals, as they are generated next to the photomultipliers: then the amount of direct light hitting the PMTs is much higher than the diffuse component resulting from reflections on the walls. The situation is inverted for primary signals. A correct reconstruction of $s 2$ is fundamental to obtain reliable localization.

An algorithm has been developed and tested on MonteCarlo-generated secondary signals [48], with the aim of reconstructing the position of the simulated pulse in the plane of the photomultipliers ( $x, y$ plane). It is essentially based on the formula used to calculate the barycentre of the light signals, starting from the relative weights of the amount of photons collected by the single PMTs. As a first precaution, an "artificial" signal has to be assigned to nonworking PMTs as, even if we do not read their signal, we must assume that photons arrive also on them. To do this, it was decided to assign to these PMTs an amount of light that resulted from the mean of the light collected by surrounding photomultipliers. After the rough position is reconstructed, two main corrections have to be applied: first, the amount of light collected by the external PMTs has to be rescaled, because it contains a significant amount of the reflected component that in an ideally infinite system would end up in farther devices. Such correction has been evaluated on its own and it has been later applied to all rings of PMTs, which receive less and less reflected light, from the outermost to the central one.

The second correction is related to the fact that, to calculate the event position, the central coordinates of the PMTs are used. Therefore, reconstructed positions tend to accumulate on these points, and there are no events outside the external ring of PMT centres. To correct this, detector slices of $5^{\circ}$ in the angular variable have been defined. For each of these slices, the correlation function between the reconstructed $\left(R_{\mathrm{rec}}\right)$ and the MC-generated $\left(R_{\mathrm{MC}}\right)$ radial positions of the events has been derived. Ideally, for perfect reconstruction, the function $R_{\mathrm{rec}}\left(R_{\mathrm{MC}}\right)$ should be a straight line with angular coefficient equal to 1 ; the use of central PMT coordinates to calculate the event position and the fact that the system is finite, however, introduce deviations in this function, especially in the outermost region. By inverting the obtained relation and applying it to the reconstructed MC data, it is possible to correct the bias in the distribution of positions, so that the dimensions and circular shape of the detector are well reproduced. The error on the reconstructed positions, evaluated on MC data after corrections, was estimated to be $\simeq 1 \mathrm{~cm}$, to be compared with the PMT minimal diameter, $4.6 \mathrm{~cm}$. In Figure 9 the distribution of errors in the reconstruction for the MC data is shown: on the left the error before corrections is displayed, on the right after corrections.

The algorithm was then applied to a sample of reanalyzed events (25000), from a run taken with $\mathscr{E}_{\text {drift }}=330 \mathrm{~V} / \mathrm{cm}$ : actual data must also be corrected for the different response of the single PMTs (in terms of phe). A small convergence on PMT centres is still visible and not removable; moreover, the reconstructed plane maintains a hexagonal-like shape (see Figure 10), which disappears in the MC data after the discussed corrections are applied. The hexagon is rotated with respect to the shape obtained before the radial correction, which is related to the PMTs positions. This is due to the reflector foils on the inner walls: the foils are fixed in six points and could have contracted during the cooling down at LAr temperature. The absence of nonworking PMTs is well visible, in Figure 10(b), for pairs 5, 11 and 31, 26, which are next to each other: in these cases regions of lower density of points are visible. PMT 33, though being on the border, is completely surrounded by working devices, and the distribution of reconstructed position clusters on its central coordinates, much like for working PMTs.

The information related to the drift coordinate $(z)$ can then be obtained from the knowledge of the drift time and velocity. The error on this value is estimated in $3 \mathrm{~mm}$, deriving from the propagation of the uncertainties on $t 1$, t2 onsets ( $\simeq 300 \mathrm{~ns})$ and on the liquid level position $(2 \mathrm{~mm})$. The uncertainty on $v_{\text {drift }}$, which was measured in $[39,40]$, is instead negligible. By adding the $z$ information, it has 


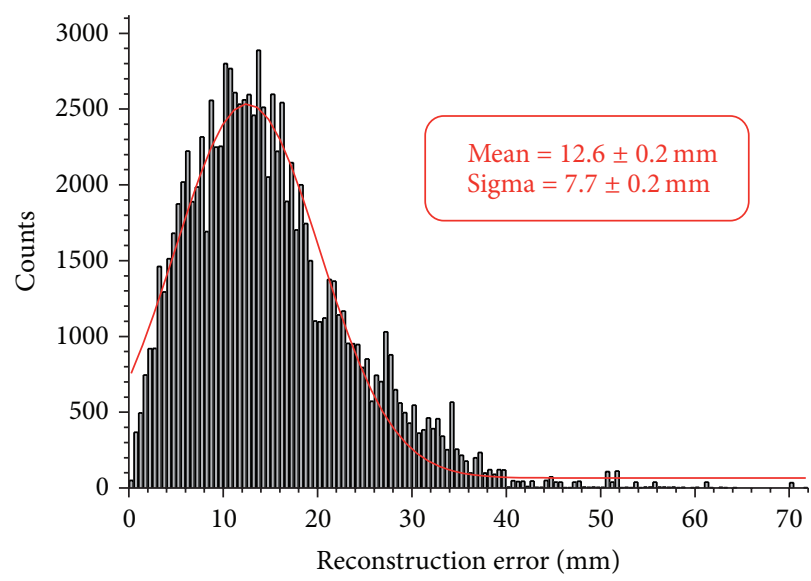

(a)

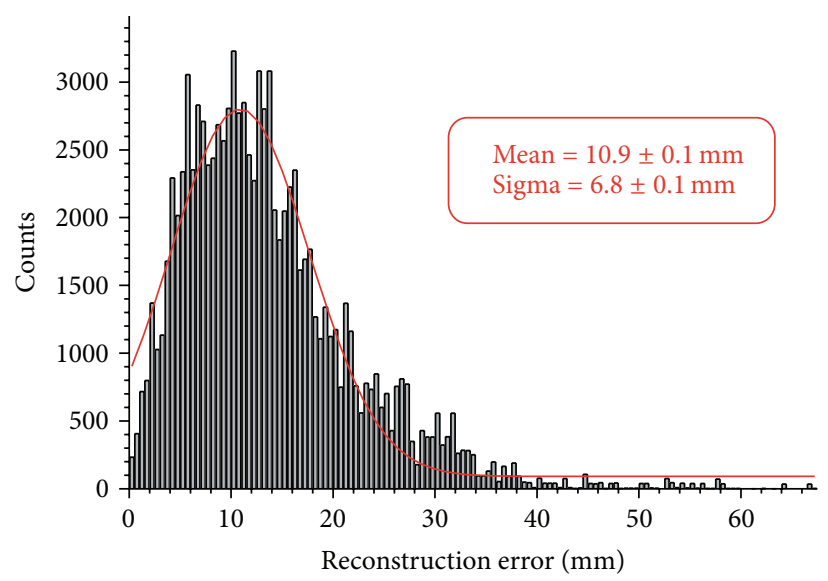

(b)

FIGURE 9: (a) Distribution of errors on reconstruction of the event position, for the sample of MC data, before corrections. (b) The same, after corrections. The error is the absolute difference between the real and reconstructed position.

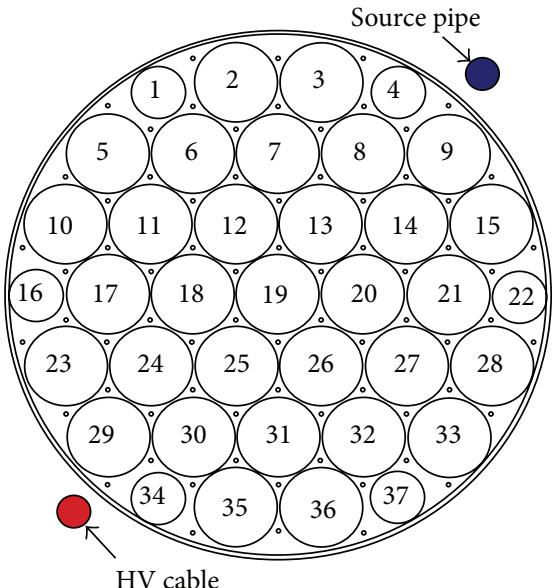

(a)

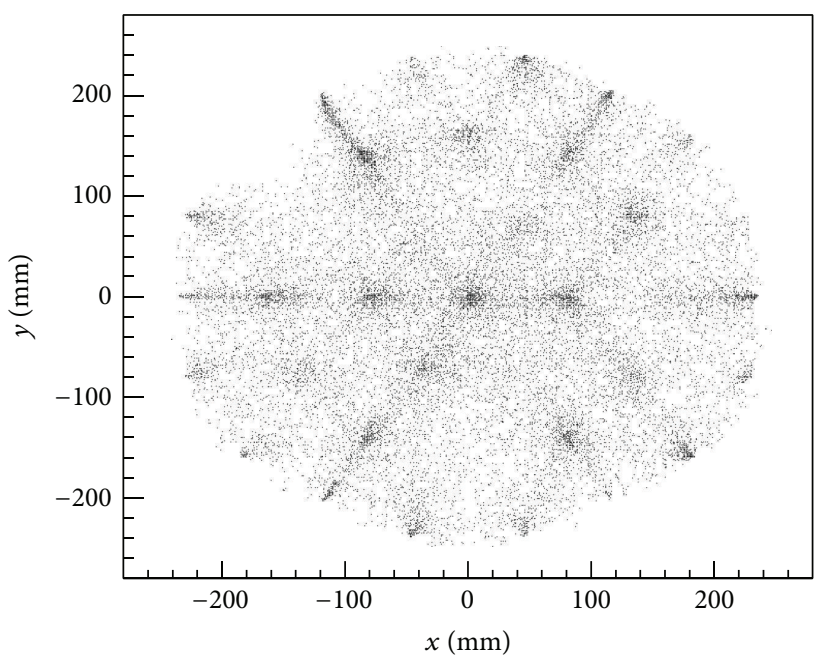

(b)

Figure 10: Reconstructed positions of 25000 events from the 2011 WArP run (b), to be compared with the actual PMT disposition (a). Nonworking PMTs are numbers 5, 11, 26, 31, and 33.

been verified that the detector profile is correctly reproduced: this is visible in Figure 11(a). In Figure 11(b) the distribution of events wrongly reconstructed outside the detector is reported, as a function of the distance from the wall. These events show a slightly larger tail than the estimated resolution for two reasons: first, the plot is a $1 \mathrm{D}$ projection of the detector external conical profile, which causes some smearing of the distribution. Secondly, external events lack, by definition, a farther outer ring of PMTs which would help calculating the correct position of the event with the adopted method: it is expected that in this boundary region errors could be slightly larger than in the inner part of the detector.

\section{Conclusions}

The WArP programme represents one of the first attempts to exploit the properties of scintillating noble liquids in double phase, as a means to discriminate $\beta / \gamma$-interactions from nuclear recoils, in order to perform dark matter searches. A powerful, years-long R\&D program, concentrated on Argon, has been carried on over the years, to study cryogenic detectors and materials and the background rejection technique based on signals produced in Argon (Sections 2.1 and 3.1). The $\mathrm{R} \& \mathrm{D}$ program led to the construction and operation of a 2.31 chamber, which demonstrated the high rejection power achievable with the mentioned techniques and was used to perform a physics run at the Gran Sasso laboratories. This was used to set limits on the WIMP $(m, \sigma)$ parameter space, excluding the DAMA positive results.

The next step of the program was a 1001 detector, whose projected sensitivity was expected to almost match present best results in the field. The detector was built and operated at LNGS, but issues related to HV distribution hindered the possibility of performing a physics run. This ultimately led to 


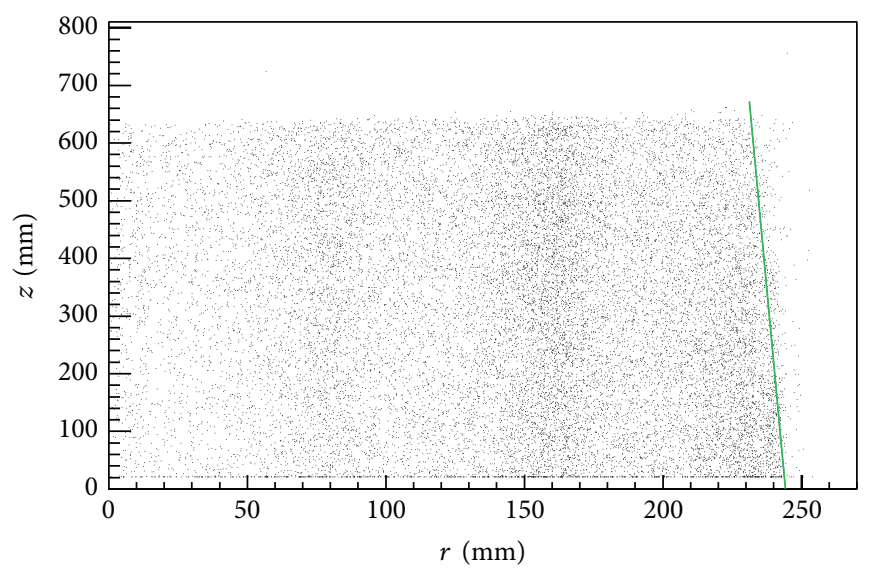

(a)

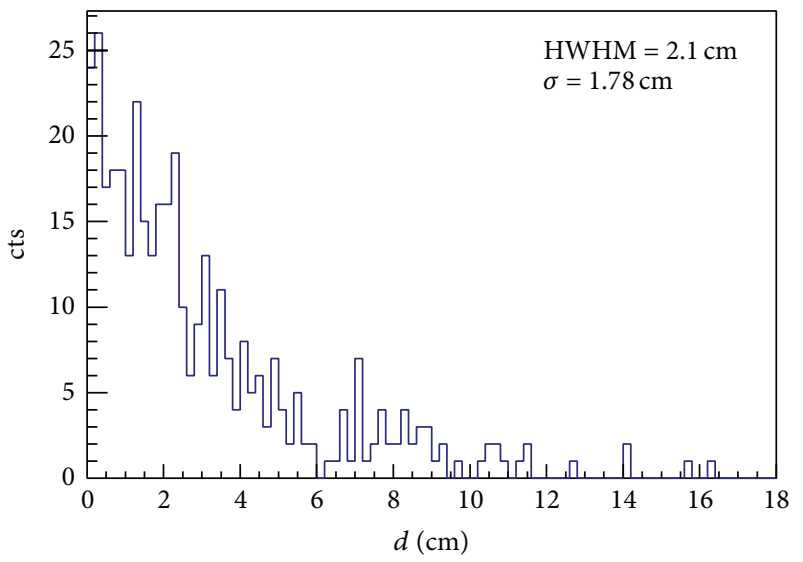

(b)

FIGURE 11: (a) Distribution of real events positions in the $(z, r)$ plane, where $z$ is the distance from the liquid level and $r$ is the reconstructed radial coordinate, as derived from $(x, y)$ information. The truncated-cone shape of the detector is well reproduced (upside-down in this view): the real slope, $s_{r}=-62$, is to be compared with the one fitted from the data, $s_{\text {rec }}=-61 \pm 1.4$. Denser regions correspond once again to the radial positions of the photomultipliers. (b) Distribution of events wrongly reconstructed outside the detector walls, as a function of their distance $d$ from the walls (details in text).

the program premature closure. Anyway in 2011 a five-month technical run was performed: data are still being analysed to characterise the detector response from a technical point of view. The analysis of the primary signals shows stability of the light collection system (PMTs), though with a measured light output lower than expectations; it is also tested the PMTs ability to recover from solicitations (due to HV problems). In the same way the analysis of secondary signals shows that they can be used to perform precise 3D localization of the events position. Preliminary analyses show that discrimination based on pulse shape analysis on primary signals can be achieved and used to partially reject online electron-induced events. Quantitative evaluation of the rejection power for the full-scale, 1001 detector is still to be performed.

Though the response of the detector in terms of collected light (Light Yield) is lower than predicted, the work carried on so far suggests that it can be a reliable instrument. Mentioned issues on $\mathrm{HV}$ stability are not related to the proposed detection technique. The technology was proven to be an effective means to perform dark matter searches (see Section 3.1); the use of Argon that couples pulse shape analysis to the $s 2 / s 1$ discrimination, also used in Xenon-based experiments, can lead to more stringent background rejection with respect to other techniques. Indeed, the implemented technology and gathered experience during the life of the WArP project can prove useful to the community for the successful outcome of other present and future LAr-based experiments, like, for example, ArDM [49] and DarkSide [50].

\section{Conflict of Interests}

The author declares that there is no conflict of interests regarding the publication of this paper.

\section{Acknowledgments}

This document summarises all the years of work and efforts by the members of the WArP Collaboration, as well as the results obtained during this period; it is written on behalf of the collaboration. The author wishes to thank the INFN for the support given to the program during its lifespan and the Gran Sasso Laboratories which made facilities and staff available during the periods of operation. This experiment started as a spinoff of the major technological advances provided by the ICARUS Collaboration, without whom this work could not have been carried on. The WArP programme has been supported since March 2006 by the National Science Foundation under Grant no. PHY-0603376. Funding from the NSF allowed developing upgraded electronics, which permitted obtaining the presented results on pulse shape discrimination. The author wishes to acknowledge the dedication and professionalism of the personnel of the sections of the INFN mechanical and electronics workshops that have designed and built the components of the 2.31 and 1001 detectors. The author also acknowledges all the people of the LNGS staff that collaborated in the construction and installation of the detectors, from the underground operations to the chemical, mechanical, and electronics services.

\section{References}

[1] Full list of Planck papers can be found at ESA site, A\&A, http://www.rssd.esa.int/index.php?project=planck.

[2] Planck Collaboration, Planck 2013 results. XVI. Cosmological parameters, 2013, http://arxiv.org/abs/1303.5076.

[3] J. Beringer, J. F. Arguin, and Particle Data Group, "Review of particle physics," Physical Review D, vol. 86, Article ID 010001, 2012. 
[4] G. Jungman, M. Kamionkowski, and K. Griest, "Supersymmetric dark matter," Physics Report, vol. 267, no. 5-6, pp. 195-373, 1996.

[5] J. D. Lewin and P. F. Smith, "Review of mathematics, numerical factors, and corrections for dark matter experiments based on elastic nuclear recoil," Astroparticle Physics, vol. 6, no. 1, pp. 87112, 1996.

[6] E. Aprile, M. Alfonsi, XENON Collaboration et al., "Dark matter results from 100 live days of XENON100 data," Physical Review Letters, vol. 107, Article ID 131302, 2011.

[7] E. Aprile et al., "Dark matter results from 225 live days of XENON100 data," Physical Review Letters, vol. 109, Article ID 181301, 2012.

[8] D. S. Akerib, H. M. Araujo, X. Bai et al., "First results from the LUX dark matter experiment at the Sanford underground research facility," Physical Review Letters, vol. 112, Article ID 091303, 2014.

[9] R. Bernabei, P. Belli, F. Cappella et al., "DAMA/LIBRA results and perspectives," Journal of Physics: Conference Series, vol. 375, Article ID 012002, 2012.

[10] CoGeNT Collaboration, C. E. Aalseth, P. S. Barbeau et al., "Results from a search for light-mass dark matter with a $p$-type point contact germanium detector," Physical Review Letters, vol. 106, Article ID 131301, 2011.

[11] CoGeNT Collaboration, C. E. Aalseth, P. S. Barbeau et al., "Search for an annual modulation in a $p$-type point contact germanium dark matter detector," Physical Review Letters, vol. 107, Article ID 141301, 2011.

[12] G. Angloher, G. M. Bauer, I. Bavykina et al., "Results from $730 \mathrm{~kg}$ ? days of the CRESST-II dark matter search," The European Physical Journal C, vol. 72, article 1971, 2012.

[13] Amerio, S. S. Amorusob, and M. Antonello, "Design, construction and tests of the ICARUS T600 detector," Nuclear Instruments and Methods in Physics Research A: Accelerators, Spectrometers, Detectors and Associated Equipment, vol. 527, no. 3, pp. 329-410, 2004.

[14] P. Benetti, E. Calligarich, R. Dolfini et al., "Dolfini Detection of energy deposition down to the $\mathrm{keV}$ region using liquid xenon scintillation," Nuclear Instruments and Methods in Physics Research A: Accelerators, Spectrometers, Detectors and Associated Equipment, vol. 327, no. 1, pp. 203-206, 1993.

[15] D. Cline, A. Curioni, A. Lamarin et al., "A WIMP detector with two-phase xenon," Astroparticle Physics, vol. 12, no. 4, pp. 373377, 2000.

[16] M. Miyajima, T. Takahashi, S. Konno et al., "Average energy expended per ion pair in liquid Argon," Physical Review A, vol. 9, article 1438, 1974.

[17] T. Takahashi, M. Miyajima, S. Konno, and T. Hamada, “The Wvalue of liquid Argon," Physics Letters A, vol. 44, no. 2, pp. 123124, 1973.

[18] J. Lindhard, V. Nielsen, and M. Scharff, "Integral equations govering radiation effects," Matematiske-Fysiske Meddelelser, Udgivet af Det Kongelige Danske Videnskabernes Selskab, vol. 33, no. 10, p. 1.

[19] J. Lindhard, V. Nielsen, and M. Scharff, "Range concepts and heavy ion ranges," Matematiske-Fysiske Meddelelser, Udgivet af Det Kongelige Danske Videnskabernes Selskab, vol. 33, no. 14, p. 1.

[20] P. J. William and J. Sadauskis, "Alpha-particle ionization in pure gases and the average energy to make an ion pair," Physical Review Letters, vol. 90, p. 1120, 1953.
[21] G. L. Cano, “Total ionization and range of low-energy recoil particles in pure and binary gases," Physical Review Letters, vol. 169, p. 277, 1968.

[22] L. Grandi, WARP: an Argon double phase technique for dark matter search [Ph.D. thesis], 2005.

[23] A. Hitachi, T. Takahashi, N. Funayama et al., "Effect of ionization density on the time dependence of luminescence from liquid Argon and xenon," Physical Review B, vol. 27, article 5279, 1983.

[24] P. Benetti, F. Calaprice, E. Calligarich et al., "Measurement of the specific activity of ${ }^{39} \mathrm{Ar}$ in natural Argon," Nuclear Instruments and Methods in Physics Research A, vol. 574, pp. 83-88, 2007.

[25] D. Acosta-Kane, R. Acciarrim, and O. Amaize, "Discovery of underground Argon with low level of radioactive ${ }^{39} \mathrm{Ar}$ and possible applications to WIMP dark matter detectors," Nuclear Instruments and Methods in Physics Research A, vol. 587, p. 46, 2008.

[26] C. Rubbia, “The liquid-Argon time projection chamber: a new concept for neutrino detectors," Tech. Rep. CERN-EP/77-08, 1977.

[27] WArP Collaboration, http://warp.lngs.infn.it.

[28] P. Benetti, R. Acciarri, WArP Collaboration et al., "First results from a dark matter search with liquid Argon at $87 \mathrm{~K}$ in the Gran Sasso underground laboratory," Astroparticle Physics, vol. 28, no. 6, pp. 495-507, 2008.

[29] WArP Collaboration, R. Acciarri, M. Antonello et al., "Effects of nitrogen contamination in liquid Argon," Journal of Instrumentation, vol. 5, Article ID 06003, 2010.

[30] R. Acciarri, M. Antonello, WArP Collaboration et al., "Oxygen contamination in liquid Argon: combined effects on ionization electron charge and scintillation light," Journal of Instrumentation, vol. 5, Article ID 05003, 2010.

[31] R. Acciarri, M. Antonello, F. Boffelli et al., "Demonstration and comparison of photomultiplier tubes at liquid Argon temperature," Journal of Instrumentation, vol. 7, Article ID 01016, 2012.

[32] F. Pröbst, "Results of CRESST phase I," Nuclear Physics B, vol. 110 , no. 2, pp. 67-69.

[33] EDELWEISS Collaboration, V. Sanglard, A. Benoit et al., "Final results of the EDELWEISS-I dark matter search with cryogenic heat-and-ionization Ge detectors," Physical Review D, vol. 71, Article ID 122002, 2005.

[34] CDMS Collaboration, D. S. Akerib, J. Alvaro-Dean et al., "New results from the cryogenic dark matter search experiment," Physical Review D, vol. 68, Article ID 082002, 2003.

[35] D. Abrams, D. S. Akerib, and M. S. Armel-Funkhouser, "Exclusion limits on the WIMP-nucleon cross section from the cryogenic dark matter search," Physical Review D, vol. 66, Article ID 122003, 2002.

[36] D. S. Akerib, J. Alvaro-Dean, M. S. Armel-Funkhouser et al., "First results from the cryogenic dark matter search in the Soudan underground laboratory," Physical Review Letters, vol. 93, Article ID 211301, 2004.

[37] A. Zani, Development and operation of large volume Liquid Argon detectors for rare events detection [Ph.D. thesis], 2013.

[38] Acciarri, R. M. Antonello, B. Baibussinov et al., "The WArP experiment," Journal of Physics: Conference Series, vol. 203, Article ID 012006, 2010.

[39] ICARUS Collaboration, S. Amoruso, M. Antonello et al., "Analysis of the liquid Argon purity in the ICARUS T600 TPC," 
Nuclear Instruments and Methods in Physics Research A: Accelerators, Spectrometers, Detectors and Associated Equipment, vol. 516, pp. 68-79, 2004.

[40] W. Walkowiak, "Drift velocity of free electrons in liquid Argon," Nuclear Instruments and Methods in Physics Research A: Accelerators, Spectrometers, Detectors and Associated Equipment, vol. 449, no. 1-2, pp. 288-294, 2000.

[41] A. Ferrari, P. R. Sala, A. Fassó, and J. Ranft, "FLUKA: a multi-particle transport code," Tech. Rep. CERN-2005-10, 2005, INFN/TC-05-11, SLAC-R-773.

[42] GEANT4 Collaboration, S. Agostinelli, J. Allison et al., "Geant4-a simulation toolkit," Nuclear Instruments and Methods in Physics Research A: Accelerators, Spectrometers, Detectors and Associated Equipment, vol. 506, no. 3, pp. 250-303, 2003.

[43] H. Wulandari, J. Jochum, W. Rau, and F. von Feilitzsch, "Neutron flux at the Gran Sasso underground laboratory revisited," Astroparticle Physics, vol. 22, no. 3-4, pp. 313-322, 2004.

[44] G. Battistoni, A. Ferrari, T. Montaruli, and P. R. Sala, "The FLUKA atmospheric neutrino flux calculation," Astroparticle Physics, vol. 19, no. 2, pp. 269-290, 2003.

[45] W. R. Leo, Techniques for Nuclear and Particle Physics Experiments, Springer, Berlin, Germany, 1987.

[46] L. Cavallero, Functional parameter analysis of the liquid Argon dark matter detector working in double-phase WArP 100l [M.S. thesis], 2012.

[47] S. Kubota, A. Nakamoto, T. Takahashi et al., "Recombination luminescence in liquid Argon and in liquid xenon," Physical Review B, vol. 17, Article ID 2762, 1978.

[48] A. Falcone, Localization of events in the WArP 100 detector [M.S. thesis], 2012.

[49] A. Badertscher, F. Bay, The ArDM Collaboration et al., "Status of the ArDM experiment: first results from gaseous Argon operation in deep underground environment," http://arxiv.org/abs/1307.0117.

[50] T. Alexander, D. Akimov, K. Arisaka et al., "Light yield in darkside-10: a prototype two-phase liquid Argon TPC for dark matter searches," http://arxiv.org/abs/1204.6218. 

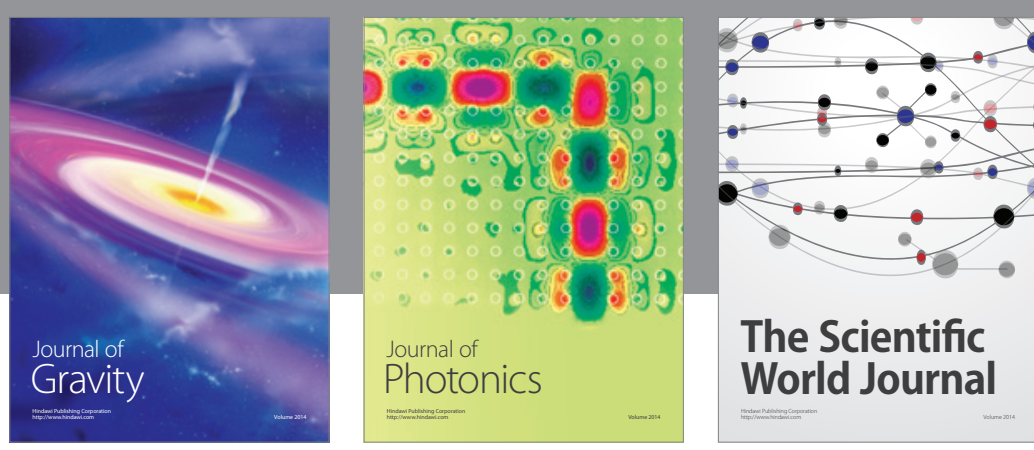

The Scientific World Journal
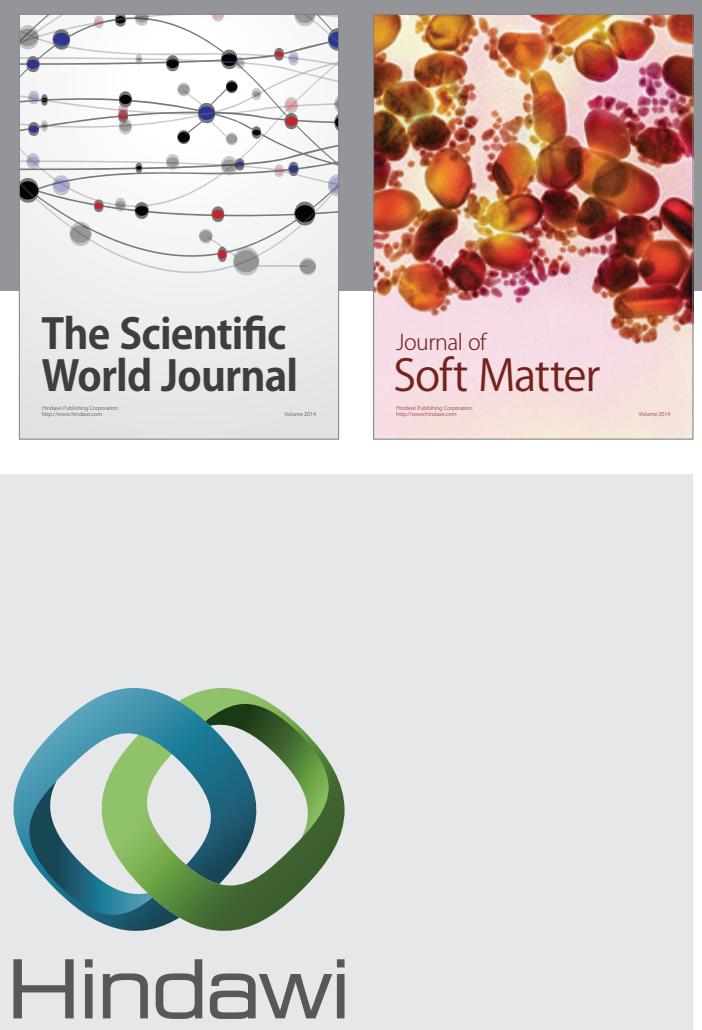

Submit your manuscripts at

http://www.hindawi.com

nternational Journal of

Statistical Mechanics
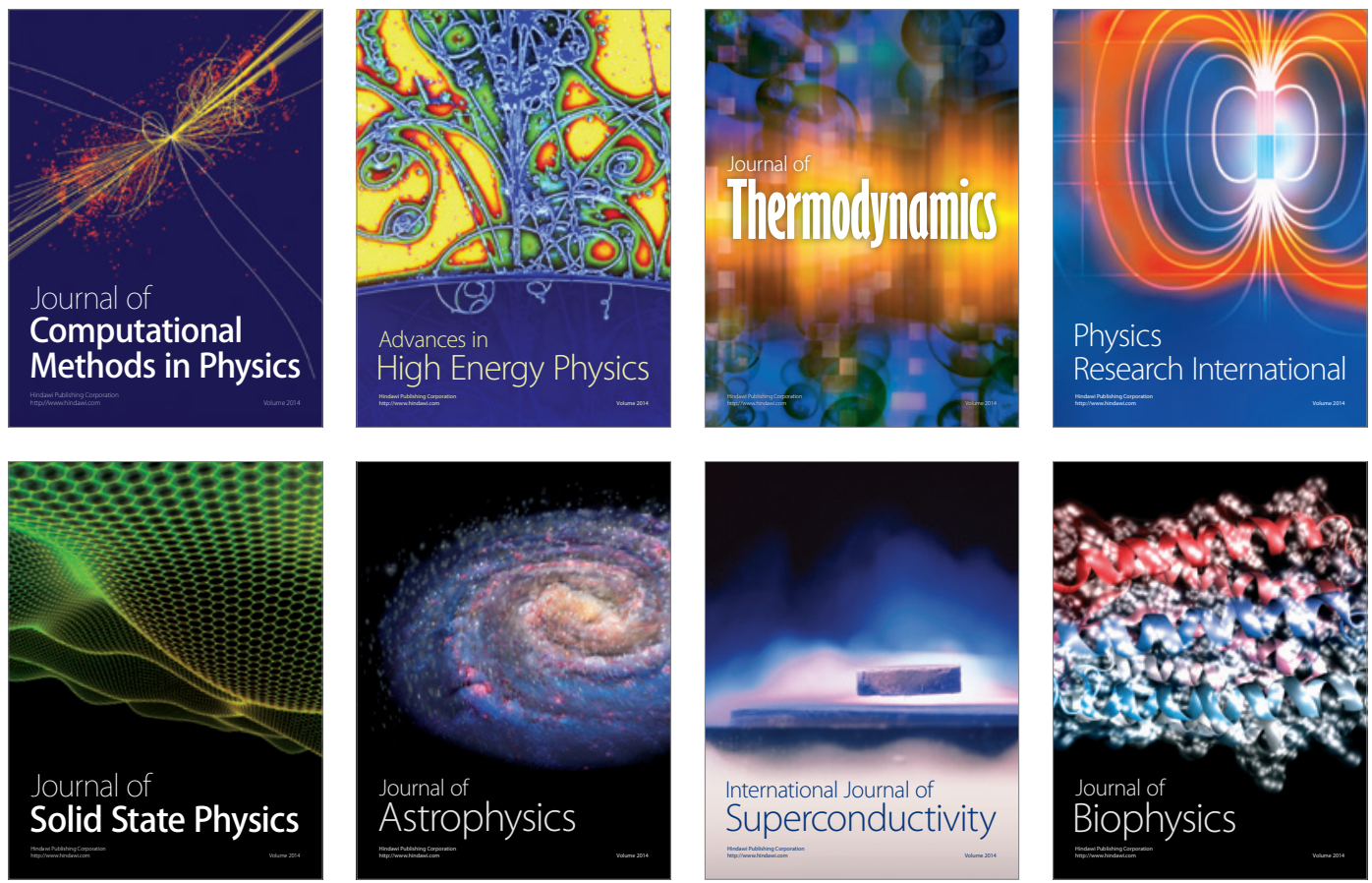
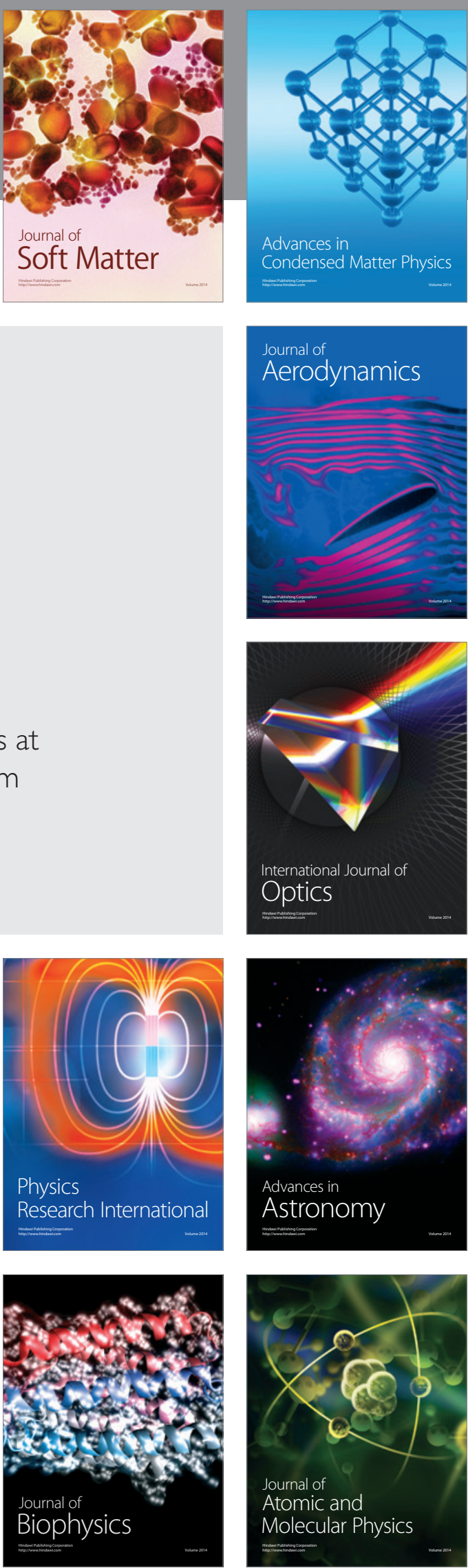